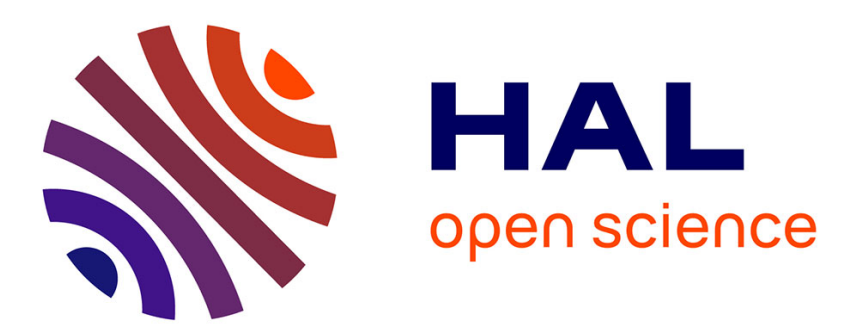

\title{
Droplet spatial distribution in a spray under evaporating and reacting conditions
}

Lola Rousseau, Christine Lempereur, Mikael Orain, Olivier Rouzaud, Olivier Simonin

\section{- To cite this version:}

Lola Rousseau, Christine Lempereur, Mikael Orain, Olivier Rouzaud, Olivier Simonin. Droplet spatial distribution in a spray under evaporating and reacting conditions. Experiments in Fluids, 2021, 62 (2), pp.26. 10.1007/s00348-020-03129-9 . hal-03317596

\section{HAL Id: hal-03317596 \\ https://hal.science/hal-03317596}

Submitted on 6 Aug 2021

HAL is a multi-disciplinary open access archive for the deposit and dissemination of scientific research documents, whether they are published or not. The documents may come from teaching and research institutions in France or abroad, or from public or private research centers.
L'archive ouverte pluridisciplinaire HAL, est destinée au dépôt et à la diffusion de documents scientifiques de niveau recherche, publiés ou non, émanant des établissements d'enseignement et de recherche français ou étrangers, des laboratoires publics ou privés. 


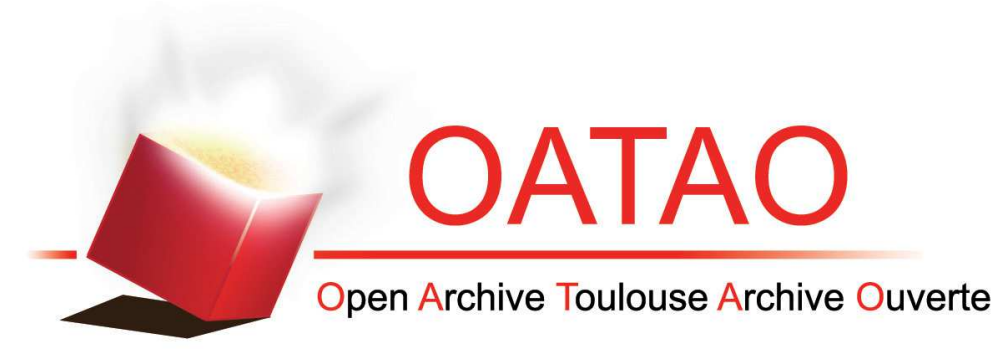

\section{Open Archive Toulouse Archive Ouverte (OATAO)}

OATAO is an open access repository that collects the work of some Toulouse researchers and makes it freely available over the web where possible.

This is an author's version published in: https://oatao.univ-toulouse.fr/28171

Official URL:https://doi.org/10.1007/s00348-020-03129-9

\section{To cite this version :}

Rousseau, Lola and Lempereur, Christine and Orain, Mikael and Rouzaud, Olivier and Simonin, Olivier Droplet spatial distribution in a spray under evaporating and reacting conditions. (2021) Experiments in Fluids, 62 (26). ISSN 0723-4864

Any correspondence concerning this service should be sent to the repository administrator: tech-oatao@listes-diff.inp-toulouse.fr 


\title{
Droplet spatial distribution in a spray under evaporating and reacting conditions
}

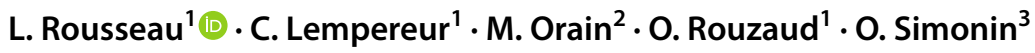

\begin{abstract}
In order to study spray combustion, an experimental test rig was developed at ONERA to partially characterize the flow conditions inside the combustion chamber of a gas turbine. Experimental campaigns using laser-based diagnostics were performed to provide an experimental database under reacting and non-reacting conditions. The paper first describes the Mie scattering image-processing to detect the droplets in the spray, and to calculate 2D maps of droplet number density and mean inter-droplet distance. The method is subsequently used to investigate the spray behavior under both reacting and non-reacting conditions according to global-averaging and phase-averaging methods. Experimental findings on the spatial droplet distribution in the spray are compared to the simple regular grid distribution and the Hertz-Chandrasekhar distribution. Results show that, under both conditions, there is an affine relationship between the inverse square root of the mean droplet number density and the nearest-neighbor inter-droplet distance. Moreover, observations suggest that the droplet spatial distribution fits more closely to a Hertz-Chandrasekhar distribution than a simple regular grid distribution, which may bring new insight for spray modeling.
\end{abstract}

L. Rousseau

lola.rousseau@onera.fr

1 ONERA, DMPE, Centre Midi-Pyrénées, Toulouse, France

2 ONERA, DMPE, Centre Midi-Pyrénées, Fauga-Mauzac, France

3 Institut Mécanique des Fluides de Toulouse (IMFT), CNRS, Université de Toulouse, Toulouse, France 


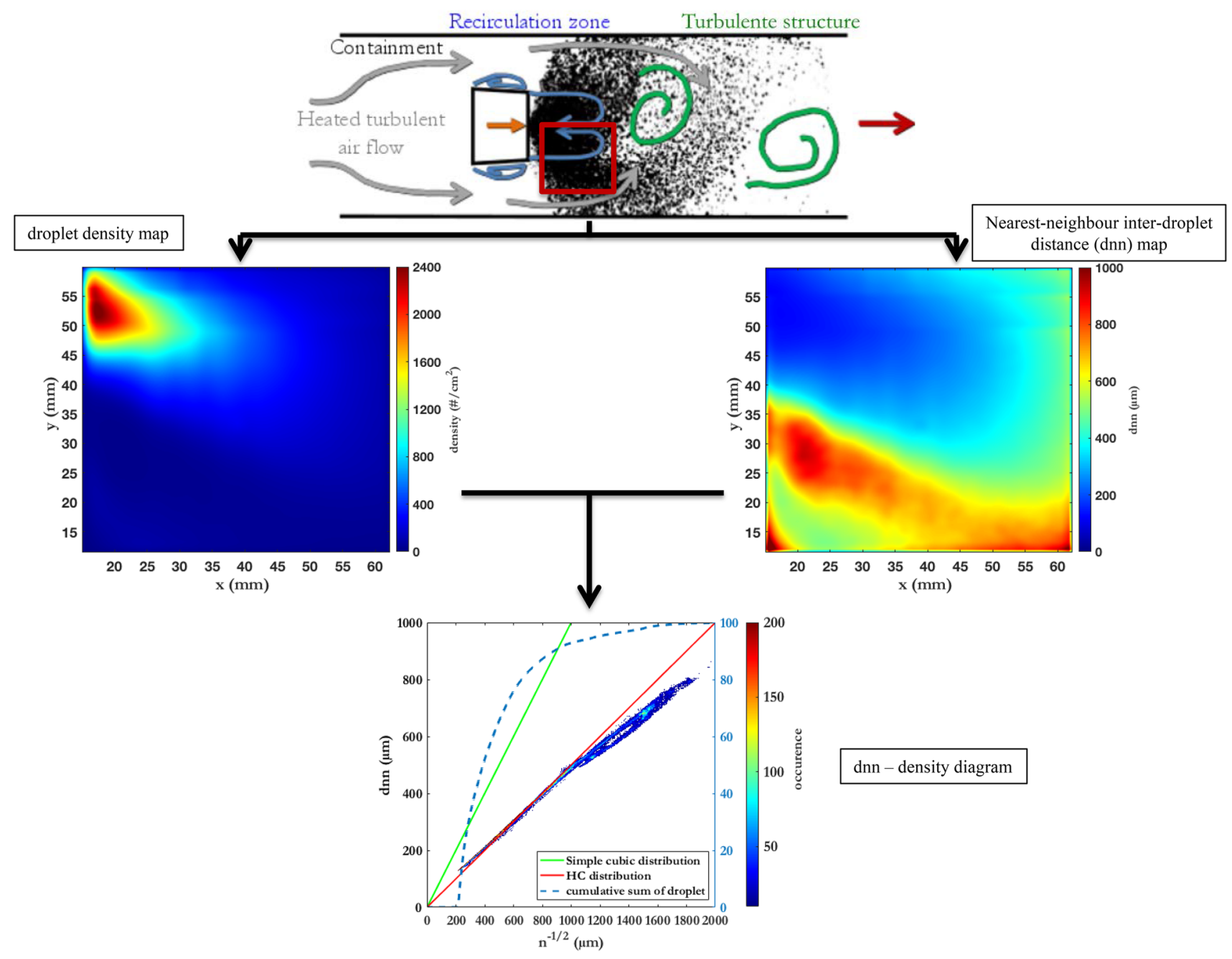

\section{Introduction}

In aeroengines, fuel is injected as a liquid into the combustion chamber in order to increase heat and mass transfer surface between fuel and oxidizer and thus improve combustion efficiency. This process involves two-phase flow turbulent combustion where the influence of atomization, droplet dispersion by turbulence and spray evaporation upon combustion processes shall be addressed, including the interactions between all these phenomena. In order to better understand spray combustion, experiments on both academic and more realistic injection systems are required. While the first studies on droplet combustion by Godsave (1953) and Spalding (1953) initially focused on the ideal case of an isolated droplet burning in a quiescent atmosphere, more complexity was gradually added to the experiments with, for example, detailed studies on monodisperse droplet streams (Labowsky 1976; Silverman et al. 1994; Orain et al. 2005), or on polydisperse sprays (Li et al. 1993; Chen et al. 1997; Mikami et al. 2009). In parallel, models were also developed in an attempt to describe more accurately spray evaporation and combustion in convective flows (Williams 1973; Abramzon and Sirignano 1989; Jiang et al. 1995; Sirignano 2014). Consequently, in the $70 \mathrm{~s}$ ' and the $80 \mathrm{~s}$ ', pioneering studies from Chiu and co-workers (Suzuki et al. 1971, Chiu et al. 1977, 1982) have shown that parameters such as droplet diameter and spacing have an influence on fuel vaporization rate and on the resulting fuel spatial distribution around the droplets. As a result, different combustion regimes can occur from individual droplet burning to the so-called group combustion. The group combustion theory suggests four combustion regimes of droplet clouds (Single droplet combustion, 
Internal group combustion, External group combustion and External sheath combustion) that are determined from the group combustion number $\mathrm{G}$ defined by:

$$
G=\frac{1.5 L e *\left(1+0.276 * S c^{\frac{1}{3}} * R e^{\frac{1}{2}}\right) * N^{\frac{2}{3}} * d}{D_{i}}
$$

where Le, Sc, Re are, respectively, the Lewis number, the Schmidt number and the Reynolds number, $\mathrm{N}$ represents the total number of droplets contained in the group, $d$ is the droplet diameter and $D_{i}$ equals the mean distance between droplet centers. In the 2000s', Chiu's theory has been revisited by Réveillon et al. (2005) who performed direct numerical simulation of dilute sprays and proposed improvements to the description of spray combustion regimes. At the same period as Chiu, Kerstein et al. (1982) developed the percolate combustion model that describes three combustion regimes for a partially premixed flow. Each regime is defined according to the following criterion:

$S=n_{v}^{\frac{1}{3}} * r_{f}=\frac{r_{f}}{\delta_{s}}$

where $n_{v}$ is the droplet number density per volume unit, $r_{f}$ is the radius of the flame which surrounds a droplet and $\delta_{s}$ is the distance between two droplets. The value of $S$ defines the combustion regime. For $S<0.41$, the configuration corresponds to a dilute spray where droplets burn as a group. As the value tends to zero, the number of droplets surrounded by the flame decreases to the point where the isolated droplet combustion regime is reached. For $S>0.73$, the configuration is that of a dense spray where pockets of gas are surrounded by flames. In the intermediate combustion regime, both previous situations occur.

It is noted that these models are principally based on a distance parameter between droplets where this distance is conventionally calculated according to the mean density of droplets in the spray. Moreover, it is also assumed that the droplets are regularly arranged in the spray, so that a simple regular grid distribution is used. In the literature, several numerical simulations and experiments based on the assumption of a simple regular grid droplet distribution have been carried out to study spray vaporization and/or combustion. For example, in the numerical study of multi-droplet arrays by Imaoka et al. (2005) and the experimental study by Chauveau et al. (2006), droplets are regularly distributed along the edges of a cube in order to analyze the influence of the inter-droplet distance and the droplet localization in the array on their evaporation and combustion. As a result, the droplet evaporation and combustion are influenced by the distance parameter, the number of droplets in the array, the ambient temperature and the droplet localization in the array. Nevertheless, the droplet distribution according to the simple regular grid arrangement is possibly an oversimplification of the real spray topology. A brief literature survey shows that the description and understanding of a cloud of particles are also relevant to a variety of fields of research. This includes, for example, meteorology, in the context of cloud formation (Kostinski et al. 2001) or astrophysics, for stellar dynamics (Chandrasekhar 1943). For this branch of astrophysics, which describes the motions of stars from the average distribution of the other distant stars, a theoretical uniform random distribution law is sometimes adopted and is called the Hertz-Chandrasekhar law (Hertz 1909; Chandrasekhar 1943). However, these models are only theoretical and, so far, few studies available in the open literature attempt to verify the assumptions of these models.

The interaction between the flow turbulence and the spatial distribution of the liquid was investigated in the recent years. For example, Squires et al. (1991) and Février et al. (2001) numerically studied the influence of turbulence on particle segregation in a turbulent flow, and Sahu et al. (2016) experimentally investigated the droplet-turbulence interactions for a polydispersed water spray. These works particularly highlight the phenomenon of preferential segregation and also show that preferential segregation does not rely on a unique turbulence scale in the flow but rather on the response time of the particle. Moreover, in order to describe preferential segregation in the flow, several methods are presented in the literature (Monchaux et al. 2012; Sahu et al. 2016) to characterize droplet clusters and voids (size, characteristic length...). For the non-reacting case, a few recent studies have focused on the interaction between the distribution of droplets in the flow and their evaporation. Amongst them are the experimental work of Cochet et al. (2009) and Sahu et al. (2018), where several laser techniques have been implemented to characterize carrier, both liquid and gaseous (fuel vapor and carrier flow) phases. In particular, the vapor spatial concentration in the flow was found to be heterogeneous and connected to the presence of droplet clusters. Therefore, in the latter study, it is interesting to note that the authors have defined the group evaporating number (based on the group combustion number of the Chiu's theory), and showed that single droplet evaporation and internal group evaporation modes are possible in this experimental configuration.

However, the majority of the literature focuses primarily isothermal flows, and similar information in reacting flows is sparse. One example is Rouzaud et al. (2016), who experimentally observed a linear relationship between the inverse square root of droplet number density in the spray and the mean inter-droplet distance in a combusting flow. Besides, the experimental data compared favorably to the $2 \mathrm{D}$ Hertz-Chandrasekhar, in terms of the mean and the standard deviation values. Therefore, it was inferred that the $3 \mathrm{D}$ 
spatial distribution of the droplet is likely to be uniformly random.

Considering the general lack of information on the spatial distribution of droplets in a spray and its connection to the turbulent structures of the flow, performing experiments to yield such correlation are necessary to further understand spray dynamics. In particular, this should help to confirm or invalidate the classical assumption of the simple regular grid distribution of droplets used in the combustion and twophase flows models. The purpose of the article is to investigate a realistic two-phase flow under reacting and nonreacting conditions by means of laser-based diagnostics in order to 1) generate an exhaustive experimental database on both liquid and gaseous phases, 2) more specifically derive 2D maps of droplet number density and mean inter-droplet distance, 3) compare the findings with typical distributions (simple cubic lattice and Hertz-Chandrasekhar) and propose possible improvements to spray models.

The remainder of the paper comprises three sections. The experimental test rig and the instrumentation used to characterize the spray behavior are first detailed. The processing algorithm developed to analyze the spray images is then described and the limitations of the method are discussed. Results for reacting and non-reacting conditions are presented and compared to simple regular grid and Hertz-Chandrasekhar distributions. The paper ends with a summary of the main findings and perspectives.

\section{Material and methods}

\subsection{Experimental test rig}

The experimental setup PROMETHEE (Fig. 1a) was developed at ONERA to represent a combustion chamber that provides flow conditions close to those encountered inside a gas turbine combustion chamber. The spray behavior can be studied under non-reacting and reacting conditions, Vicentini (2016). Only a brief description of the rig, installed on the ONERA LACOM test facility, is recalled here. It is composed of five main parts: air and fuel supplies, a flow generator, a combustion chamber, an injector and a fuel exhaust. Air is supplied from a high-pressure tank connected to a $1 \mathrm{MW}$ electrical heater that can deliver a mass flow rate of up to $1 \mathrm{~kg} . \mathrm{s}^{-1}$ when heated up to a maximum temperature of $900 \mathrm{~K}$. Air mass flow rate is measured with a sonic nozzle located downstream of the electrical heater. Liquid kerosene is pressurized in a tank and supplied to the test rig by means of a fuel line equipped with a Coriolis flow controller (in the range 0-10 g.s ${ }^{-1}$ ). The flow generator consists of conditioners (section transformation from a circular to a square shape) together with several grids, which allow generating controlled turbulence. The combustion chamber is composed of a watercooled sector and has an internal square section equal to $120 \times 120 \mathrm{~mm}^{2}$. In order to allow non-intrusive optical measurements, the combustion chamber is equipped with UV-transparent windows for laser access and visualization purposes. A trapezoidal bluff-body, with a $42 \%$ blockage ratio, is located at the chamber inlet in the span-wise direction and leads to the development of a recirculation

(a)

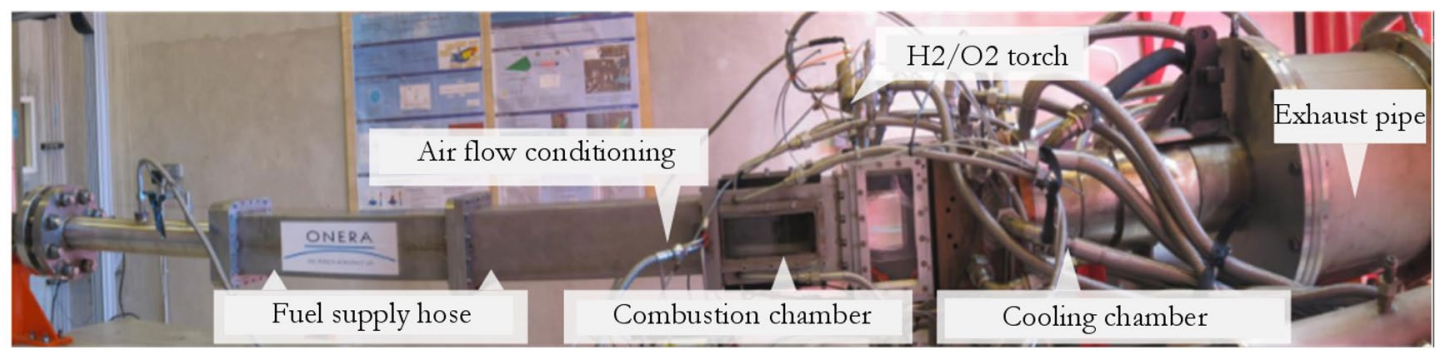

(b)

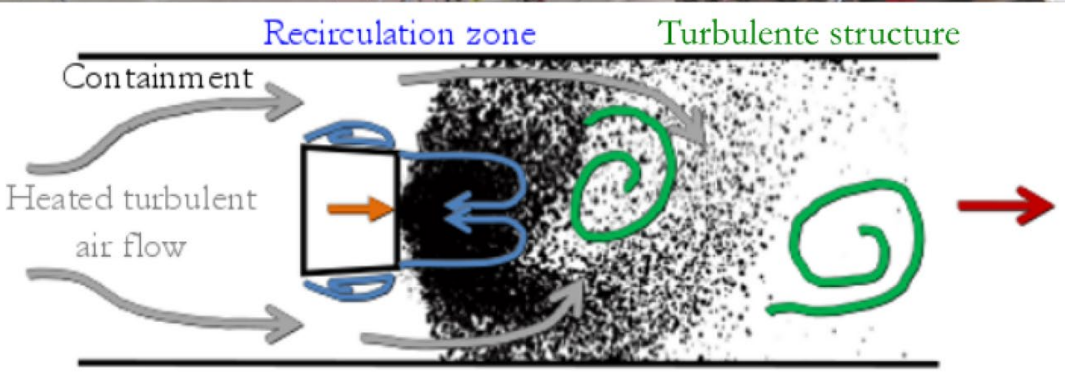

Fig. 1 a View of the experimental PROMETHEE test rig $\mathbf{b}$ sketch of the combustion chamber 
Fig. 2 Sketch of the injector included in the PROMETHEE test rig

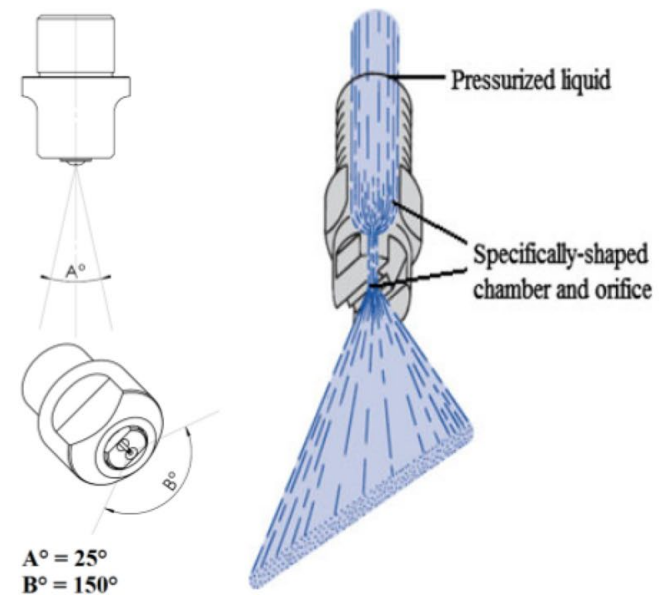

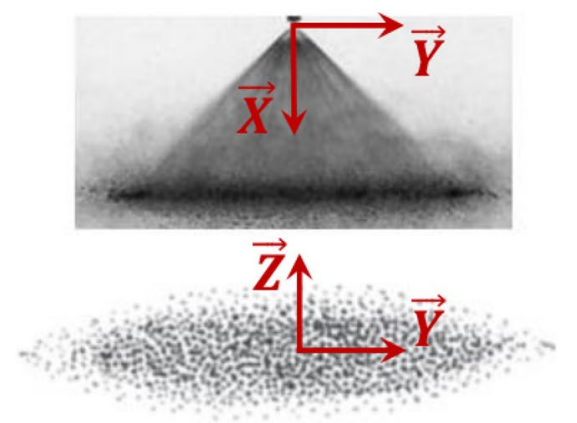

zone downstream of the bluff-body. Under non-reacting conditions, a vortex shedding phenomenon occurs in the combustion chamber (Fig. 1b). The fuel injection system consists of a flat-fan nozzle (Fig. 2) fixed at the rear of the bluff-body, on its axis, which creates an elliptical-shaped polydisperse spray of droplets. The ignition of the fuel-air mixture is achieved by a hydrogen-oxygen torch. Finally, the burnt gases flow into the exhaust pipe where they are cooled down by means of water injection.

The incoming air flow is at standard atmospheric pressure and its temperature is equal to $450 \mathrm{~K}$. Liquid fuel injected in the combustion chamber is $n$-decane ( $95 \%$ purity) and its initial temperature is $330 \mathrm{~K}$. Fuel and air mass flows are, respectively, equal to $1 \mathrm{~g} . \mathrm{s}^{-1}$ and $58 \mathrm{~g} . \mathrm{s}^{-1}$ and the global equivalence ratio is around 0.24 . The mean flow velocity is equal to $5.8 \mathrm{~m} . \mathrm{s}^{-1}$, such that the Reynolds number based on the height of the bluff-body is about 22,000 .

\subsection{Measurement techniques}

With the aim of better understanding spray evaporation and combustion and to validate numerical simulations, it is necessary to create an exhaustive experimental database. To this end, several optical measurement techniques are implemented on the PROMETHEE experimental setup to obtain a variety of data about the two-phase flow in the combustion chamber under both non-reacting and reacting conditions. Particle Image Velocimetry (PIV) is used to characterize velocity field of the gaseous phase. The liquid phase characterization is based on Phase Doppler Anemometry (PDA) and Mie scattering images, which allows to measure droplet velocity and diameter, and spatial distribution of droplets in the spray. Planar Laser-Induced Fluorescence applied to $\mathrm{OH}$ radical (OH-PLIF) and chemiluminescence emission from $\mathrm{OH}^{*}$ (where * indicates that the radical is in an excited electronic state) are used to locate and investigate the flame front for the reacting conditions. In this paper, we will only present the results from Mie scattering images.

Planar Mie scattering measurements are performed using a Nd:YLF laser (from Quantronix) together with a Phantom V341 high-speed camera. The laser wavelength is $527 \mathrm{~nm}$, its energy is equal to $10 \mathrm{~mJ}$ per pulse, and the duration of the laser pulse is about $200 \mathrm{~ns}$. The laser beam is transformed into a light sheet that illuminates the droplet spray in the combustion chamber and Mie scattering from droplets is collected by the high-speed camera at $90^{\circ}$ from the laser sheet propagation. The camera is equipped with a $1800 \times 1600$ pixel $^{2}$ array. The dynamic range of the camera is 0-4095 counts (12-bit digitization). The field of view represents an area of $51 \times 46 \mathrm{~mm}^{2}$, so that the spatial resolution is roughly $29 \mu \mathrm{m}$ per pixel.

The size of the visualized flow area is deliberately chosen smaller than the height of the combustion chamber in order to optimize the spatial resolution of the images, which leads to a more accurate description of the flow phenomena. As a result, either the upper part of the flow or its lower part is visualized by the cameras (Fig. 3).

In order to identify flow instabilities under non-reacting conditions, a differential pressure measurement is implemented by means of pressure transducers (from Validyne) located on the upper and the lower walls of the bluff-body.

\subsection{Data collection}

Several tests have been conducted under both non-reacting and reacting conditions.

For non-reacting conditions the data are collected over 3830 raw Mie scattering images, which cover the upper part of the combustion chamber (gray area in Fig. 3). Additionally, the signal of the differential pressure located on the walls of the bluff-body is simultaneously recorded. For these conditions, the pressure signal is periodic, which confirms 
Fig. 3 Location of Mie scattering and OH-PLIF images with respect to the injector body

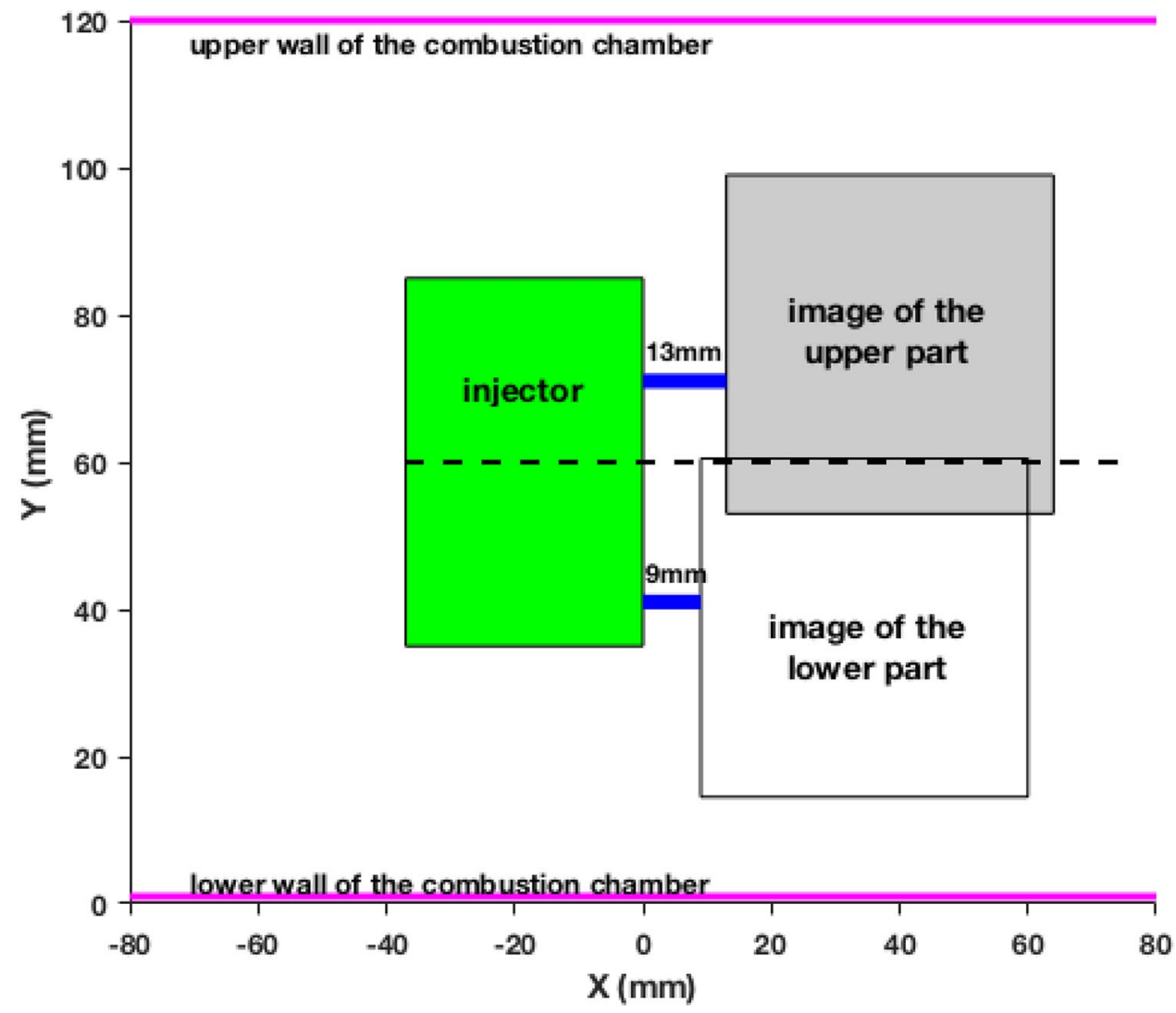

the apparition of a vortex shedding phenomenon, also named Von Karmàn vortex street, behind the bluff-body.

For reacting conditions, each part of the combustion chamber (shown in gray and white in Fig. 3) have been visualized and each set of data contains 3830 raw Mie scattering images and the differential pressure signal. As expected (Bailly et al. 1996; Lovett et al. 2011), the signal of differential pressure on the bluff-body is no longer periodic, confirming that von Karmàn vortex streets disappear with combustion.

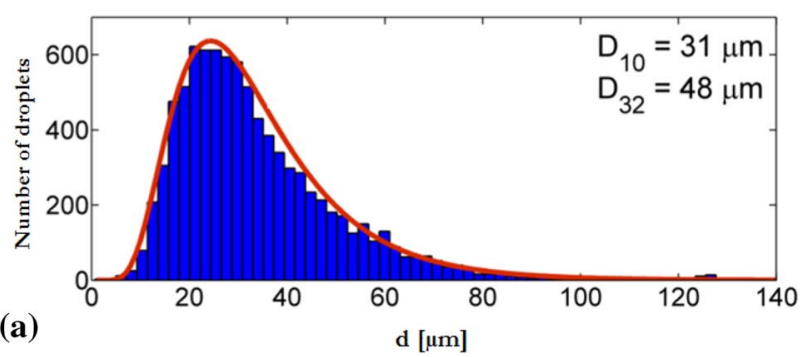

\section{Data processing of Mie scattering images}

\subsection{Optical issues}

Before image processing can be performed, it is necessary to assess the ability of the technique to detect droplets, according to the spray characteristics and to the optical configuration.

First, a preliminary analysis of the droplet intensity dynamics must be done. A typical diameter distribution, obtained by PDA measurements at $X=9 \mathrm{~mm}, Y=60 \mathrm{~mm}$ from the injector, is shown in Fig. 4a. A distribution with a similar span but with a flatter profile is also measured

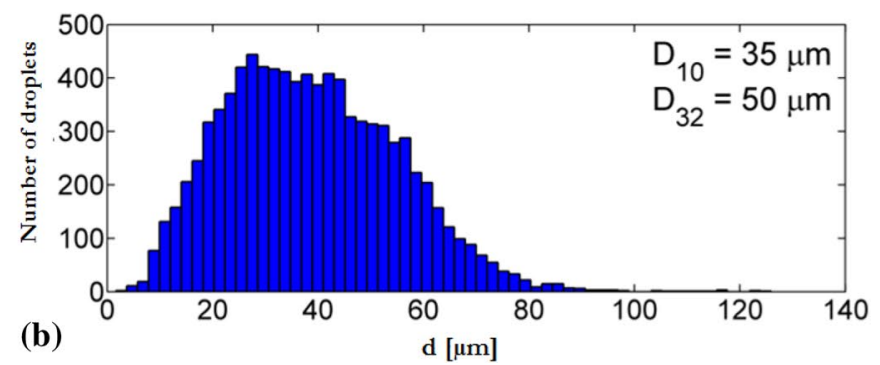

Fig. 4 Droplet diameter distributions for $\mathbf{a} X=9 \mathrm{~mm} Y=60 \mathrm{~mm} \mathrm{~b} X=53 \mathrm{~mm} Y=60 \mathrm{~mm}$ 
at $X=53 \mathrm{~mm}$ and $Y=60 \mathrm{~mm}$ (Fig. 4b). In both cases, the droplet diameter ranges from 10 to $80 \mu \mathrm{m}$ and the scattered light intensity of the largest droplets is thus 30 times higher than that of the smallest ones, according to a Mie plot* (Laven 2018) calculation taking into account the refractive index of the liquid, the wavelength of the laser and the scattering angle. As the spatial resolution is close to $29 \mu \mathrm{m} / \mathrm{px}$, the smallest particles are imaged on one pixel, whereas the largest ones spread on the neighboring pixels (the image diameter results from the convolution of the Point Spread Function of the imaging system with the geometric image of the particle). In any case, since the largest particles are close to saturation on the image, the smallest ones can be detected with a 12-bit digitization (4095 levels) without losses.

Second, it is well known that multiple scattering which occurs in dense sprays generates unwanted stray light on the images and may bias intensity-based measurements (Grosshans 2015). Hence, an estimation of the optical depth of the spray region is necessary. Following the Beer-Lambert's law, light intensity I decreases exponentially as it propagates through an absorbing/scattering medium:

$I(x)=I(0) \exp (-O D)$

with OD the optical depth locally defined on the geometrical path x as $O D=N \pi Q_{e x t} r^{2} x . Q_{e x t}$ is the extinction efficiency factor, $r$ is the mean droplet radius and $N$ represents the number of droplet per volume unit.

In the current experiments, the optical depth was estimated from the droplet radius $r$ and number density $N$ provided by Phase Doppler Anemometry measurements. The extinction efficiency factor Qext is close to 2 in this diameter range. The light transmission through a vertical $Y$ profile remains higher than $37 \%(\mathrm{OD}<1)$ in the major parts of the spray, so that these regions can be considered as optically dilute, thus corresponding to the single light scattering regime. Nevertheless, close to the injector, the optical density can reach as high as 5 near the axis, but this region is spatially limited and out of the scope of the image. Moreover, a close inspection of the images shows that the droplets can be easily detected and no significant blur is visible in between the droplets. Since the parameters of interest are geometrical ones only (i.e., droplet location), no significant blurring effects due to multiple scattering alter the detection algorithm.

In dense sprays $(\mathrm{OD}>>1)$, droplet count errors may occur due to overlapping droplet images and to a reduced contrast between the images and the blurred background. In such cases, structured illumination such as SLIPI (Mishra 2014) may help improve the technique capabilities by giving higher image contrast and revealing individual droplets, which in turn subsequently reduces the limitations of the detection algorithm.

Finally, the depth of field of the technique is limited to the thickness of the laser sheet, roughly $1 \mathrm{~mm}$, but out-of-field droplets can be present on the images. An « off-field» big droplet can have a similar scattering signal than a small droplet in the field and in the laser sheet. Nonetheless, since the technique does not intend to relate Mie scattering intensity to droplet size, but aims only at determining geometrical parameters (droplet position and inter-distance), the results of the detection algorithm are not affected by out-of-field droplets.

\subsection{Algorithm}

In order to characterize the liquid phase in the flow, Mie scattering images were processed to: evaluate droplet number density and nearest-neighbor inter-droplet distance (named dnn throughout the paper) fields; and also to study the correlation between these two values of interest. To this end, an image-processing algorithm was developed in MATLAB $®$ to handle Mie scattering image sets recorded in non-reacting as well as in reacting conditions. This algorithm is structured into two mains parts: the first one allows to extract the droplets from the image background and derive their location in the flow, the second one is used to calculate droplet number density and mean dnn from these pre-processed data.

First, a dewarping operation is performed on each image to correct perspective projection effects and present images in the world coordinates of the laser sheet. A ratio of $25 \mu \mathrm{m}$ per pixel was chosen for the dewarped images. An example of Mie scattering image is shown in Fig. 5a with a zoom on a moderately dense area. Second, a combination of morphological dilation and image arithmetic operations is applied to better segregate droplets and extract their contours (Fig. 5b). A disk-shaped structuring element is used for this procedure: its radius is fixed at 3 pixels in order to avoid droplet merging. Third, from the gray-level image of the droplet contours, a binarization operation is applied with the aim of highlighting droplets from the background. The binarization threshold must be carefully chosen in order to retain the smallest droplets. A parametric study about the influence of this threshold on droplet detection was carried out, and a value of 150 (in the dynamic range 0-4095) is retained as a good trade-off between droplet detection and noise suppression as will be shown later. Finally, blob analysis is performed to compute statistics for connected regions in this binary image: labeling functions are applied to identify all the droplets and locate them with their barycenter. At the end of this main step of the processing algorithm, a barycenter map (Fig. 5c) is created for each image. It is important to notice 

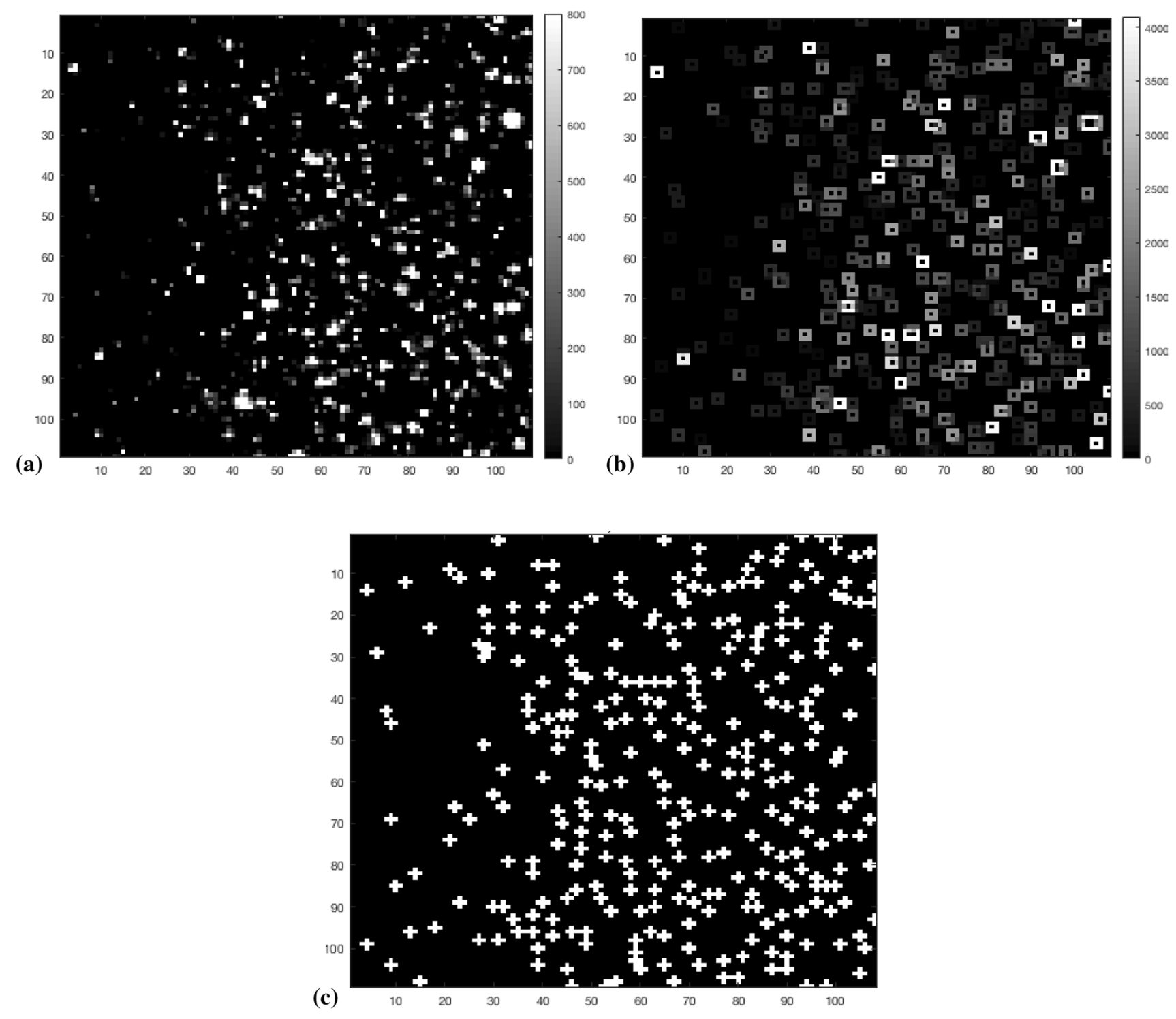

Fig. 5 Zoom $(100 \times 100$ px $)$ on an image for each step of the Mie scattering image-processing algorithm: a dewarped Mie scattering image, $\mathbf{b}$ image of droplet contours, $\mathbf{c}$ barycenter map for a threshold of 150

that the droplets are of varying brightness on an uneven background and, in dense regions, are quite close to each other, making the task of segmentation more challenging.

While Eq. 2 deals with droplet number density per volume unit, the current measurements provide droplet number density per surface unit. The influence of 3D effects will be detailed later in the paper (end of IV.1). Information about density of droplets in the flow and distance between droplets can be determined from the barycenter map. Droplet number density is a geometrical parameter which corresponds to the number of droplets per area relative to the pixel $(\mathrm{x}, \mathrm{y})$. It is locally calculated over a square area (M x M pixels $\left.{ }^{2}\right)$, called metapixel, and centered on the pixel $(\mathrm{x}, \mathrm{y})$. Thus, the mean droplet number density for the pixel $(\mathrm{x}, \mathrm{y})$ of image " $\mathrm{i}$ " is defined as follows:

$\overline{n(x, y)}^{i}=\frac{O R_{\text {meta } P x}(x, y)^{i}}{S_{\text {meta } P x}}$

where $O R_{\text {metaPx }}(x, y)^{i}$ is the metapixel occupancy ratio for the image " $i$ " which means the number of droplets located within the metapixel centered on the pixel (x,y) and $S_{\text {metaPx }}$ is the surface of the metapixel.

The value $d n n^{i}(x, y)$ represents the smallest distance between a droplet " $d$ " located at the pixel $(\mathrm{x}, \mathrm{y})$ in the i-th image and its nearest neighbor " $d$ ". It is defined as the Euclidian distance: 
$d n n^{i}(x, y)=\sqrt{\left(x_{d}-x_{d^{*}}\right)^{2}+\left(y_{d}-y_{d^{*}}\right)^{2}}$

and $d n n^{i}(x, y)$ is arbitrarily chosen equal to zero when there is no droplet present at pixel (x,y).

Similarly to the mean droplet number density, the mean dnn for the pixel $(x, y)$ in the image " $i$ " is calculated over the metapixel and is obtained as:

$\overline{\operatorname{dnn}(x, y)}^{i}=\frac{1}{O R_{\mathrm{meta} P x}(x, y)} * \sum_{k=-\frac{M}{2}}^{\frac{M}{2}} \sum_{w=-\frac{M}{2}}^{\frac{M}{2}} d n n^{i}\left(x_{k}, y_{w}\right)$

After calculating these values of interest on each pixel for the $N_{i}$ images of a specific set, an arithmetic averaging is performed over all of the images. Thus, the mean droplet number density and the mean dnn for a pixel $(x, y)$ are defined as follows:

$\bar{n}(x, y)=\frac{1}{N_{i}} \sum_{i=1}^{N_{i}} \overline{n(x, y)}^{i}$

$\overline{d n n}(x, y)=\frac{1}{N_{i}} \sum_{i=1}^{N_{i}} \overline{\operatorname{dnn}(x, y)}^{i}$

The standard deviation of the dnn is calculated in the same way: Fig. 6a and b show an example of mean droplet number density and mean dnn maps, respectively. The $\mathrm{x}$-axis represents the distance from the injector outlet and the y-axis the distance from the lower wall of the combustion chamber.

In the reacting case, a linear relationship between the inverse square root of mean droplet number density $\bar{n}(x, y)$ and the mean nearest-neighbor inter-droplet distance $\overline{d n n}(x, y)$ and its standard deviation $\sigma_{d n n}(x, y)$ has been previously obtained (Vicentini 2016; Rouzaud et al. 2016) and can be written as:

$\left\{\begin{array}{l}\overline{d n n}(x, y) \propto \alpha * \bar{n}(x, y)^{-\frac{1}{2}} \\ \sigma_{d n n}(x, y) \propto \beta * \bar{n}(x, y)^{-\frac{1}{2}}\end{array}\right.$

where $\alpha$ and $\beta$ are the corresponding dimensionless mean and standard deviation of the nearest-neighbor inter-droplet distance distribution. In the theoretical case of the simple regular grid distribution where the droplets are regularly arranged, $\alpha$ equals 1 and $\beta$ equals 0 . In the case of the $2 \mathrm{D}$ Hertz-Chandrasekhar distribution, $\alpha$ is equal to 0.50 and $\beta$ is equal to 0.26 . For details of the equations, please refer to the appendix. Experimental values obtained by Vicentini (2016) for $\alpha$ and $\beta$ are, respectively, equal to 0.59 and 0.36 .

Based on this observation, a scatter plot showing the evolution of the mean dnn and mean standard deviation versus the mean droplet number density (also referred to as a dnn-droplet number density diagram) is generated for a set of images under non-reacting or reacting conditions. Figure 7 shows an example of this type of diagram. Colors are assigned to each point according to its occurrence. Points with an occurrence lower than 10 are not shown. The dimensionless mean and standard deviation of the simple regular grid model and the 2D Hertz-Chandrasekhar (HC) one are, respectively, represented by the green line and the red line. The blue dashed line is the cumulative number of droplets expressed in percentage of the total number of droplets in the images.
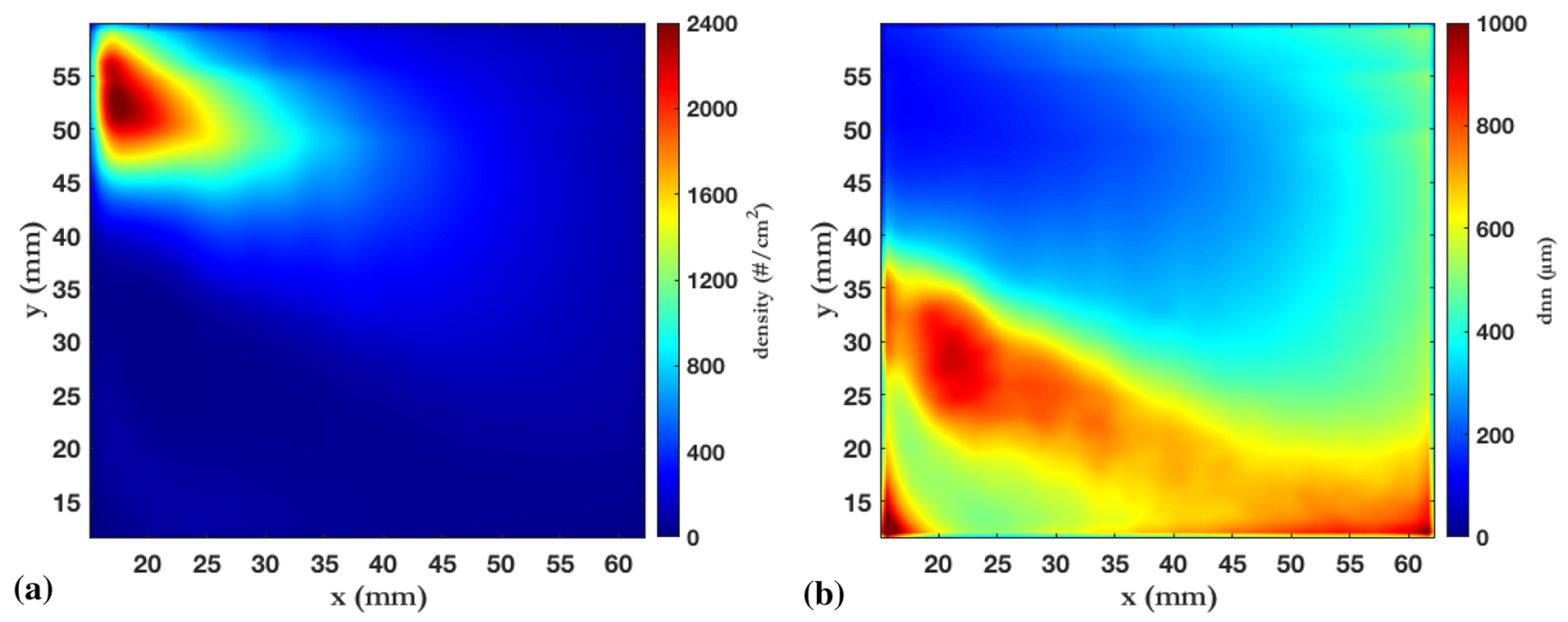

Fig. 6 Spatial distribution of a mean droplet number density and $\mathbf{b}$ mean dnn, for reacting conditions with a threshold of 150 and a metapixel size of $75 \times 75$ for the lower part of the combustion chamber. See Fig. 3 for the position 


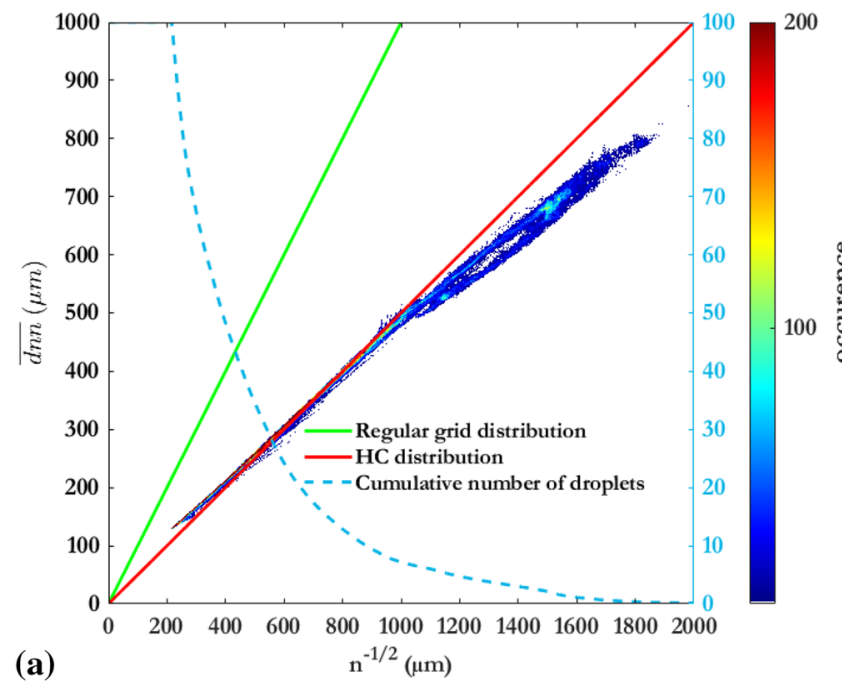

Fig. 7 dnn-droplet number density diagram for reacting conditions in the lower part of the spray. a mean dnn $\mathbf{b}$ standard deviation of dnn. The red line represents the Hertz-Chandrasekhar distribu-

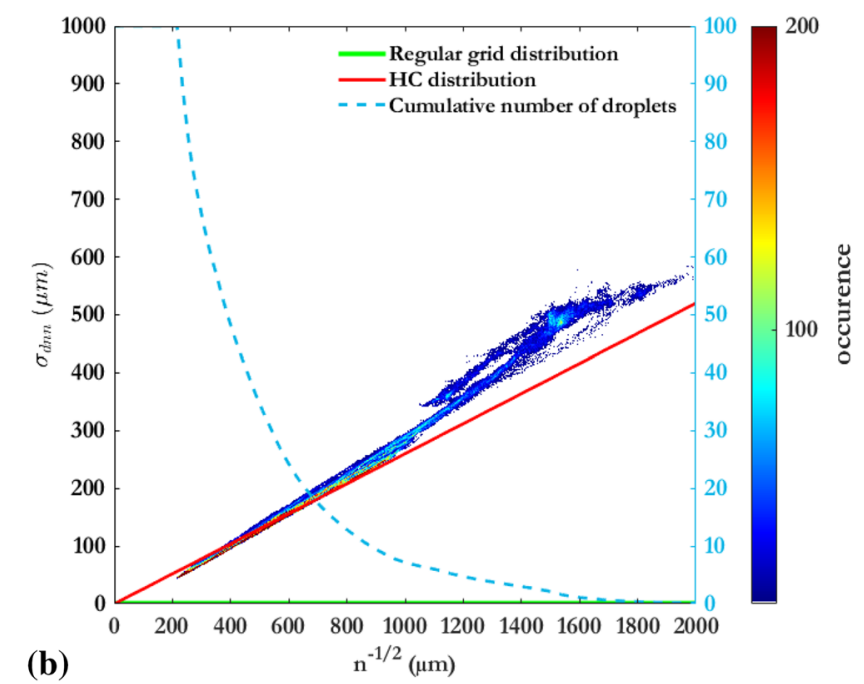

tion and the green one the simple regular grid distribution. The blue dashed line is the cumulative number of droplets expressed in percentage of the total number of droplets in the images
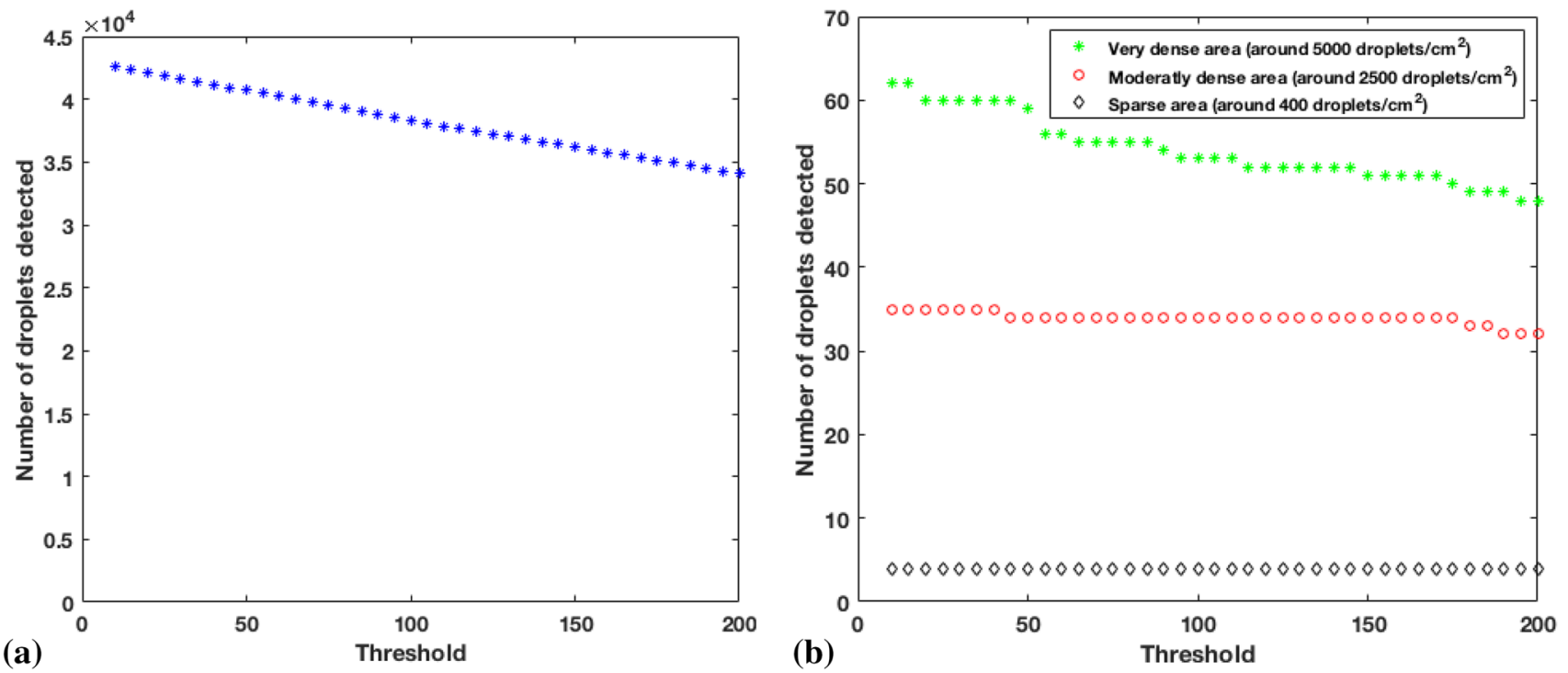

Fig. 8 Evolution of the number of droplets detected on a Mie scattering image as a function of the binarization threshold for non-reacting conditions: $\mathbf{a}$ on the global image and $\mathbf{b}$ on specific areas of $35 \times 35$ pixels $^{2}$

\subsection{Influence of the binarization threshold on droplet detection}

In a second step, the influence of the binarization threshold must be studied. The processing algorithm of Mie scattering images uses a threshold which can be chosen between 0 and 4095 and has a significant impact on droplet detection. On one hand, if the threshold value is too low, there is a risk of false detection due to residual noise on the image. On the other hand, a large number of valid droplets may be eliminated when using a high threshold value (Fig. 8a).
The evolution of the number of droplets detected on three areas of different densities as a function of the binarization threshold is shown in Fig. 8b. The number of droplets steadily decreases with the threshold, especially in the denser region, whereas it remains stable in sparse regions. This observation could be explained by the fact that small droplets have already been vaporized far away from the injector, where the droplet number density is low. For this reason, in the densest areas, a lower the threshold results in the improved detection of the small droplets. However, from the observation of the images, we decided that a pixel 

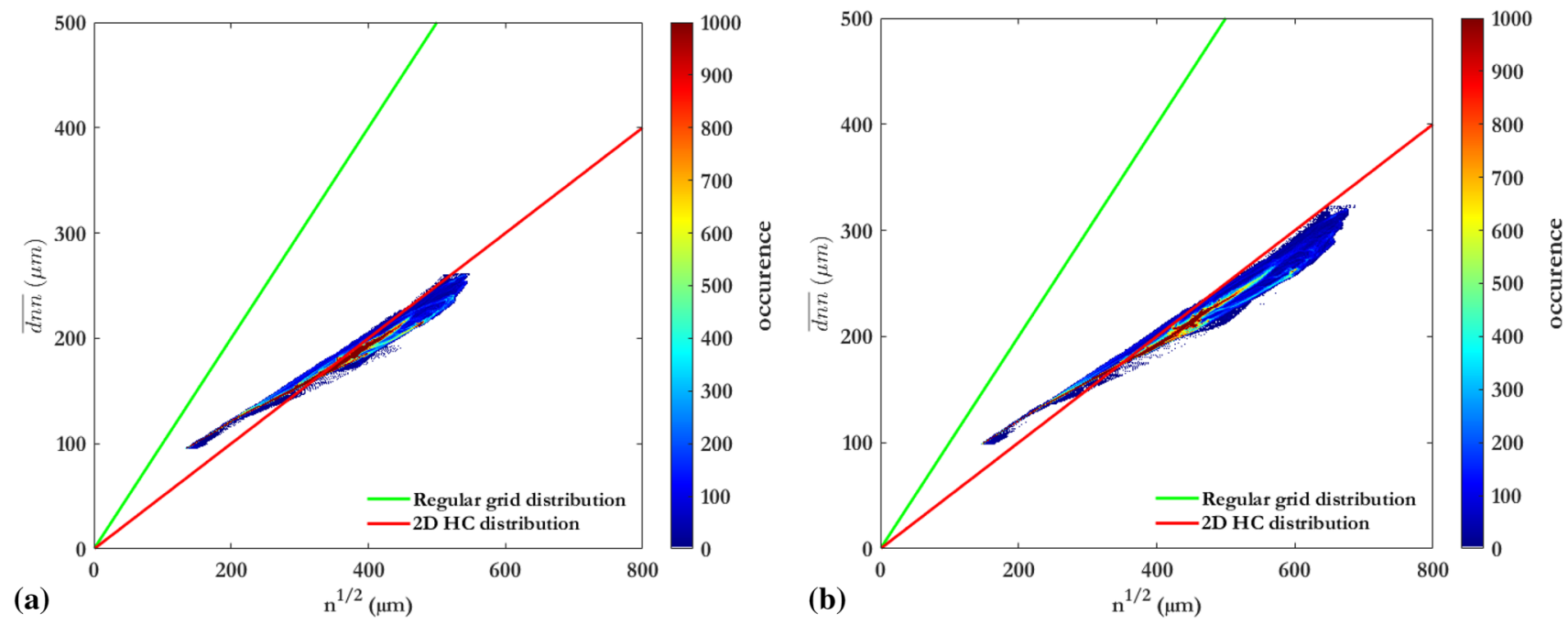

Fig. 9 Influence of the threshold on dnn-droplet number density diagram for non-reacting conditions with a metapixel size of $75 \times 75$ and a threshold value of a 150 and b 400

with a value lower than 100 ( $2.5 \%$ of the dynamic range) corresponds to noise or to defocused droplets located on the rims of the laser sheet. Consequently, a threshold between 100 and 200 seemed satisfactory and the value was set to 150. In such images where droplets can be smaller than the surface imaged by a pixel, a droplet count error is inevitable and must be quantified: in a typical image recorded in non-reacting flow conditions, 36,200 droplets are detected with a threshold of 150 , while around 38,300 and 34,100 droplets are detected with a threshold value of 100 and 200, respectively. Therefore, the error on the number of droplets detected is estimated to be $\pm 6 \%$ within this threshold range.

The binarization threshold influences the number of droplets detected and consequently the correlation between droplet number density and mean dnn (Fig. 9). For example, for a threshold equal to 150 , the maximum dnn value is around $250 \mu \mathrm{m}$ while for a threshold equal to 400 , the maximum dnn value is around $310 \mu \mathrm{m}$. Fewer droplets are detected with a higher threshold which implies larger distances between droplets and lower droplet number density: the scatter plot is thus expanding toward the highest values of $d n n$ and $\frac{--\frac{1}{2}}{n}$. However, the linear relationship between the inverse square root of droplet number density and mean dnn remains independent of the threshold. The linear regression between the mean values of dnn and droplet number density taking into account all the occurrences in the diagram provides an $\alpha$ value estimated at 0.37 when the threshold is equal to 150 and 0.38 for a threshold of 400 . Similarly, the $\beta$ values given by linear regression are, respectively, equal to 0.38 and 0.39 .

\subsection{Influence of the metapixel size on values of interest}

As mentioned before, for a pixel $(\mathrm{x}, \mathrm{y})$, droplet number density and mean dnn are calculated over a metapixel which size must be chosen as a compromise between noise reduction and smoothing of values of interest. Obviously, a small metapixel leads to noisy maps, whereas a large metapixel makes the image smoother. Figure 10 shows the mean dnn-droplet number density diagram as a function of the metapixel size $\left(25 \times 25\right.$ pixels $^{2}, 75 \times 75$ pixels $^{2}$ and $201 \times 201$ pixels $^{2}$ ). Similarly to the effect observed with the binarization threshold, the linear relationship between the inverse square root of droplet number density and mean dnn remains independent of the metapixel size. The three metapixel sizes $\left(25 \times 25\right.$ pixels $^{2}, 75 \times 75$ pixels $^{2}$ and $201 \times 201$ pixels $\left.^{2}\right)$ provide comparable dimensionless mean $\alpha$ value around 0.35 . In order to have a sufficient number of droplets in the metapixel and a 2D-map of droplet spatial distribution with enough details, a metapixel size of $75 \times 75$ pixels $^{2}$ is chosen for the results presented in the remainder of this paper, corresponding to an area of $1.8 \times 1.8 \mathrm{~mm}^{2}$ on the images.

These results (III.2 and III.3) are particularly important as they show that the fine tuning of the image-processing parameters has little impact on the mean dimensionless $\alpha$ value of dnn-droplet number density graph. In the remainder of the paper, the figures will present the results obtained for a threshold value equal to 150 and a metapixel size equal to $75 \times 75$. 

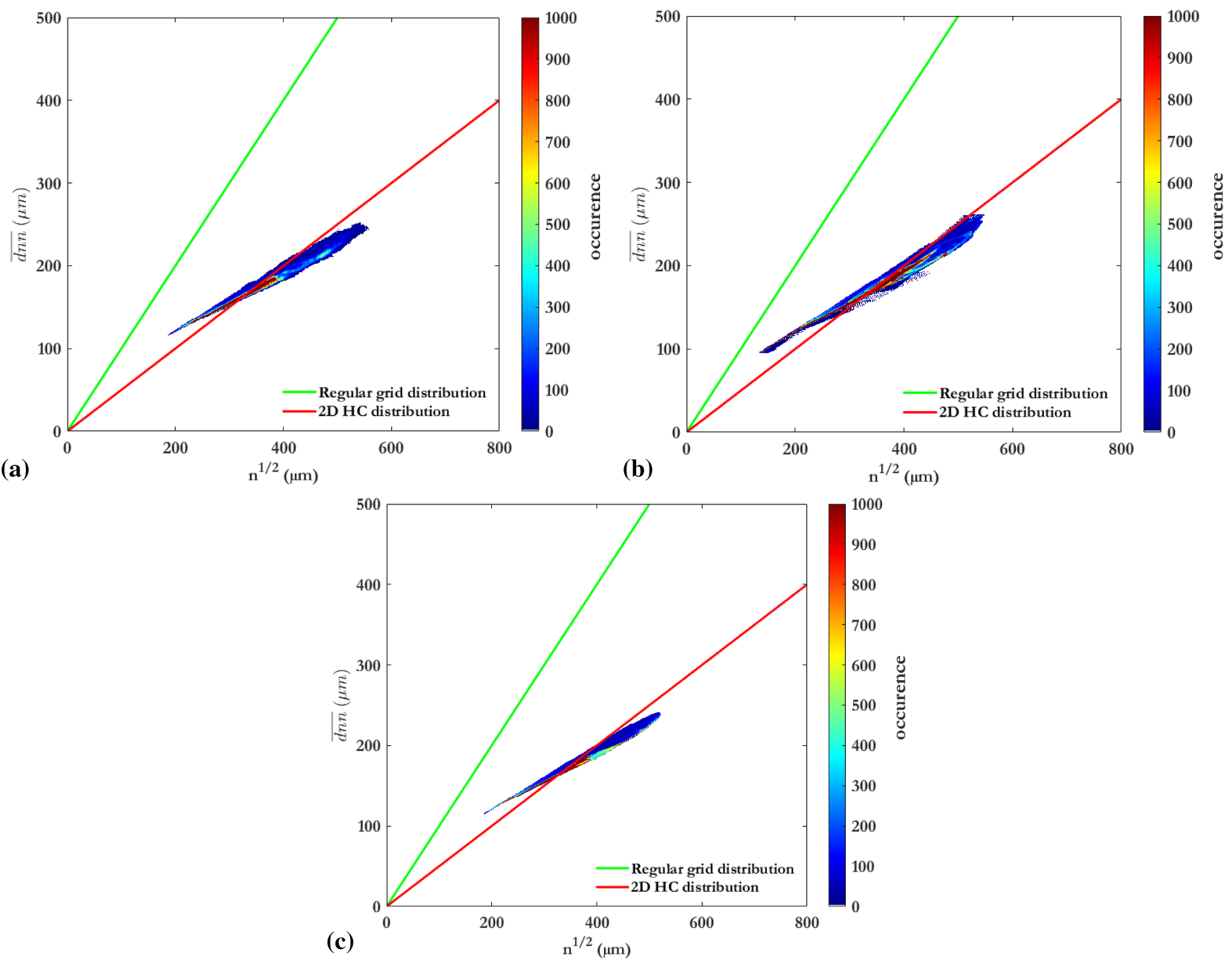

Fig. 10 Influence of the metapixel size on dnn-droplet number density diagram for non-reacting conditions, with a threshold of 150 and a metapixel size of a $25 \times 25$, b $75 \times 75$ and $\mathbf{c} 201 \times 201$

\subsection{Data averaging method}

Mie scattering images provide instantaneous information about droplet spatial distribution. Nonetheless, for a better understanding of flow phenomena, it is useful to derive statistical information, for example mean values of droplet number density and inter-droplet distance, standard deviation, etc. In the previous section, Fig. 6 shows the mean values of droplet number density and dnn from the average over the 3830 images. This processing is called "global averaging" in the following sections, and is used to process images obtained under reacting conditions.

However, as already mentioned, a von Karmàn vortex street occurs in the combustion chamber under non-reacting conditions, leading to a periodic differential pressure signal between the lower and the upper walls of the bluff-body. It is then interesting to use this signal as a reference in order to sort the whole set of Mie scattering images with respect to time and to perform phase-locked analysis. Applying a Fourier transform to this periodic signal, from which the mean value was subtracted, provides the main signal frequency of the vortex shedding. It is then necessary to determine the zero crossings of the signal after applying a Butterworth filter centered on the main frequency. The agreement between image acquisition time and zero crossings serves to allocate a specific phase to each image of the whole set. Finally, the phase space has to be discretized in a limited number of bins with an interval length $\Delta \Phi$. The choice of this value is a trade-off between time accuracy and a sufficient amount of data to perform statistically converged averaging. Vicentini (2016) has studied the influence of this value on the phase averaging when processing PIV images obtained with the current experimental setup. The vortex shedding frequency was around $33 \mathrm{~Hz}$ and a value of $\Delta \Phi$ between $4^{\circ}$ and $6^{\circ}$ was sufficient to obtain consistent results. This processing is called phase averaging in the following sections and a 
value of $4^{\circ}$ is retained for $\Delta \Phi .85$ images are used in order to calculate the mean and standard deviation images for each of the 8 phases studied $\left(0^{\circ}, 45^{\circ}, 90^{\circ}, 135^{\circ}, 180^{\circ}, 225^{\circ}, 270^{\circ}\right.$ and $315^{\circ}$ ).

\section{Results and discussion}

\subsection{Spray behavior under reacting conditions}

Figure 6 shows the spray behavior under reacting conditions where the mean droplet number density map (Fig. 6a) ranges from 0 to 2400 droplets per square centimeter and the mean dnn map (Fig. 6b) ranges from 0 to $1000 \mu \mathrm{m}$. Several observations can be made on these figures. As expected, the spray density is not homogeneous in the combustion chamber. The highest droplet number density area is located close to the outlet of the injector and near its axis (upper left corner of the droplet number density map) with a maximum value around 2400 droplets per square centimeter. Near the injector axis, the droplet number density gradually decreases as the distance to the injector increases: for example droplet number density values around 1400 and 200 droplets per square centimeter can be observed at $(X=25 \mathrm{~mm}, Y=55 \mathrm{~mm})$ and ( $X=50 \mathrm{~mm}, Y=55 \mathrm{~mm}$ ), respectively. Similarly, the droplet number density decreases far away from the injector axis with, for example, a value of 2000 droplets per square centimeter at $(X=15 \mathrm{~mm}, Y=50 \mathrm{~mm})$, while it is around 450 droplets per square centimeter at $(X=15 \mathrm{~mm}, Y=40 \mathrm{~mm})$. This can be partially explained by droplet evaporation and combustion. Finally, by comparing Fig. 6a and b, as expected, the smallest values of mean dnn (blue regions) correspond to spray regions with a high droplet number density and, by contrast, regions with a low droplet number density have high values of mean dnn.

The mean and standard deviation of dnn-droplet number density diagrams are plotted in Fig. 7a and b, respectively. Several conclusions can be drawn from these diagrams. Firstly, similarly to the results from Vicentini (2016) and Rouzaud et al. (2016), a correlation can be found between $d n n\left(\right.$ resp. $\left.\sigma_{d n n}\right)$ and $\frac{-1}{n}$. However, these authors used a simplified image processing compared to the one implemented here. Furthermore, they derived the relationship from only six regions of interest in the Mie images, while we perform statistics over the entire area of these images. Additionally, in the present paper, a regression taking into account all the occurrences in the diagram is calculated in order to have a more accurate estimate of the $\alpha$ and $\beta$ values. Two regions can be observed. In the first part corresponding to a droplet number density higher than 100 droplets $/ \mathrm{cm}^{2}\left(n^{-\frac{1}{2}}<1000\right)$, the cloud of points is almost perfectly aligned on the random 2D Hertz-Chandrasekhar distribution, with a dimensionless mean equal to 0.46 . In the second part, for lower densities ranging from 100 down to 30 droplets $/ \mathrm{cm}^{2}$, the $\alpha$ value is lower (0.36) but still close to the 2D Hertz-Chandrasekhar distribution. The dispersion of the scatter plot increases in this part of the graph: at a given droplet number density corresponds a larger range of dnn values. This observation could result from a statistical effect because there are fewer droplets (thus fewer samples) in low-droplet number density regions or from a droplet clustering effect, which implies smaller dnn than the average. However, the cumulative number of droplets indicates that only $7 \%$ of the total number of droplets is addressed in the second part of the graph which confirms the fact that the droplet distribution actually fits the 2D Hertz-Chandrasekhar distribution. A sensitivity study indicates that this result does not depend on the threshold used for droplet detection. This is a very important finding, because numerical simulations of spray vaporization and/or combustion usually consider a simple regular grid distribution of droplets. Current results suggest that existing modeling may need to be adapted in order to account for a more realistic spatial distribution of droplets in sprays. Firstly, it is noted that the relative difference between the $\alpha$ value given by the 2D Hertz-Chandrasekhar model and the experimental data is equal to $8 \%$ for a droplet number density higher than 100 droplets $/ \mathrm{cm}^{2}$ and $28 \%$ for lower values. Secondly, it is possible to estimate the relative error on the mean dnn between experiments and modeling approaches. It is around $50 \%$ with the simple regular grid distribution and between $0.5 \%$ and $5 \%$ with the 2D Hertz-Chandrasekhar one. Similar results are expected for the non-reacting case. Moreover, the dimensionless standard deviation experimental value is around 0.33 , which is larger than the one given by the $2 \mathrm{D}$ Hertz-Chandrasekhar model $(\beta=0.26)$.

As already mentioned in the ILASS2016 paper (Rouzaud et al. 2016), it is interesting to notice that such a discrepancies for the $\alpha$ and $\beta$ values may be associated with the $2 \mathrm{D}$-projection effect of the three-dimensional droplet distribution by the experimental process. As a matter of fact, by applying the same experimental process to a three dimensional uniformly random particle distribution given by a Monte-Carlo simulation, Rouzaud et al. showed that this process leads to a nearest-neighbor inter-droplet distance mean and standard deviation larger than the ones of the 2D Hertz-Chandrasekhar model, in particular due to the 2D projection effect. Since the behavior of the 2D experimental data and the 2D projected Monte-Carlo data were closed, it was inferred that the experimental three-dimensional spatial distribution of the droplets might be very close to the uniformly random distribution given in the frame of the 3D Hertz-Chandrasekhar model. This discrepancy could also be explained by the preferential segregation effects which tend to decrease the $\alpha$ value and increase the $\beta$ value (Vicentini 2016; Boutsikakis 2020). 
It is noted that the minimum value of the mean interdroplet distance is close to $140 \mu \mathrm{m}$ in Fig. 7. Actually, two 'one-pixel' droplets could be detected as close as a two-pixel distance but droplets are generally larger than a pixel on the image, which implies a minimum distance of 4 to 5 pixels, i.e., $125 \mu \mathrm{m}$. No experimental data can be obtained below this point $\left(d n n=140 \mu \mathrm{m}, \bar{n} \approx 2750\right.$ droplets $/ \mathrm{cm}^{2}$ ).

The same graphs are calculated for the upper part of the spray: they lead to similar conclusions and the dimensionless mean and standard deviation are very close to the ones obtained in the lower part. The $\alpha$ value of the first part is indeed equal to 0.43 while that of the second part is equal to 0.41 and the value for $\sigma_{d n n}$ is around 0.35 .

\subsection{Spray under non-reacting conditions}

As expected, the spray density is higher (and the mean dnn values are lower) for non-reacting conditions than for reacting conditions because the vaporization rate is lower without combustion. Under non-reacting conditions, the highest droplet number density in the spray is around 5300 droplets per square centimeter, whereas the droplet number density is reduced to around 2400 droplets per square centimeter under reacting conditions. Similarly, the maximum mean dnn in the spray under non-reacting conditions in the same area of the combustion chamber is around $350 \mu \mathrm{m}$, whereas the maximum value for reacting conditions is around $1200 \mu \mathrm{m}$.

The mean droplet number density map and the mean dnn map for the phases $\left(0^{\circ}, 45^{\circ}, 90^{\circ}, 135^{\circ}, 180^{\circ}, 225^{\circ}, 270^{\circ}\right.$ and $315^{\circ}$ ) are obtained with the phase averaging method, but

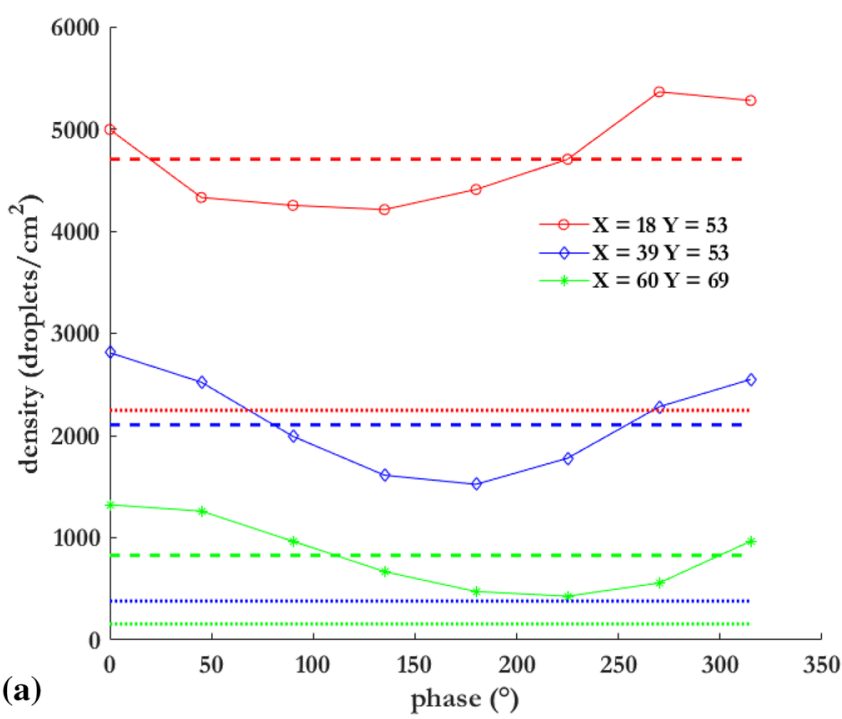

Fig. 11 Evolution of $\mathbf{a}$ the mean droplet number density and $\mathbf{b}$ the mean dnn as a function of the phase for three locations in the flow (value calculated over a metapixel with a size of $75 \times 75$ and a threshold of 150). The dashed lines represent the value obtained with the are not shown here for the sake of brevity. Similar to reacting conditions, for all the phases, the mean spray density is higher close to the injector than at large axial distances. For example, for the phase $0^{\circ}$, the mean droplet number density is over 4500 droplets per square centimeter at $(X=15 \mathrm{~mm}$, $Y=54 \mathrm{~mm})$, whereas it is around 1350 at $(X=58 \mathrm{~mm}$, $Y=54 \mathrm{~mm}$ ). Again, this can be partially explained by droplet evaporation but also by a high level of turbulence which tends to spread droplets over a large area. Finally, a spray flapping motion due to von Karmàn vortex shedding is captured with the phase averaging. At $0^{\circ}$, part of the spray is located in the upper area of the combustion chamber. Between $0^{\circ}$ and $135^{\circ}$, the spray moves down and reaches its lowest position in the combustion chamber for $135^{\circ}$ where only a small part of the spray is visible. Then, the spray moves up, and is roughly aligned with the injection axis at $270^{\circ}$, to finally reach its highest position in the chamber at around $315^{\circ}$. In order to refine these observations, Fig. 11 shows the evolution of the mean droplet number density and dnn according to the phase for different locations in the spray. Red, blue and green colors are assigned to pixels located, respectively, at position $(X=18 \mathrm{~mm}, Y=53 \mathrm{~mm})$, $(X=39 \mathrm{~mm}, Y=53)$ and $(X=60 \mathrm{~mm}, Y=69 \mathrm{~mm})$, dashed lines represent the mean value calculated with the global averaging at each location and dotted lines represent the mean value calculated for the reacting case at each location. The evolution of the mean droplet number density and mean dnn may be compared to a semi-periodic evolution for each metapixel, which confirms the flapping motion due to the von Karmàn vortex shedding. Furthermore, for each

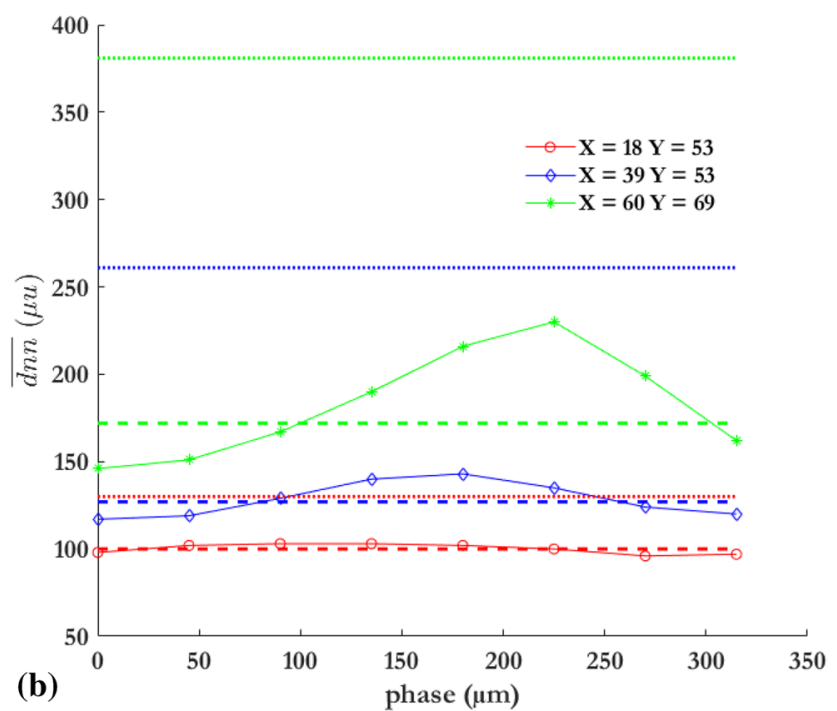

global averaging at each location. The dotted lines represent the value obtained with the global averaging at each location for the reacting case 


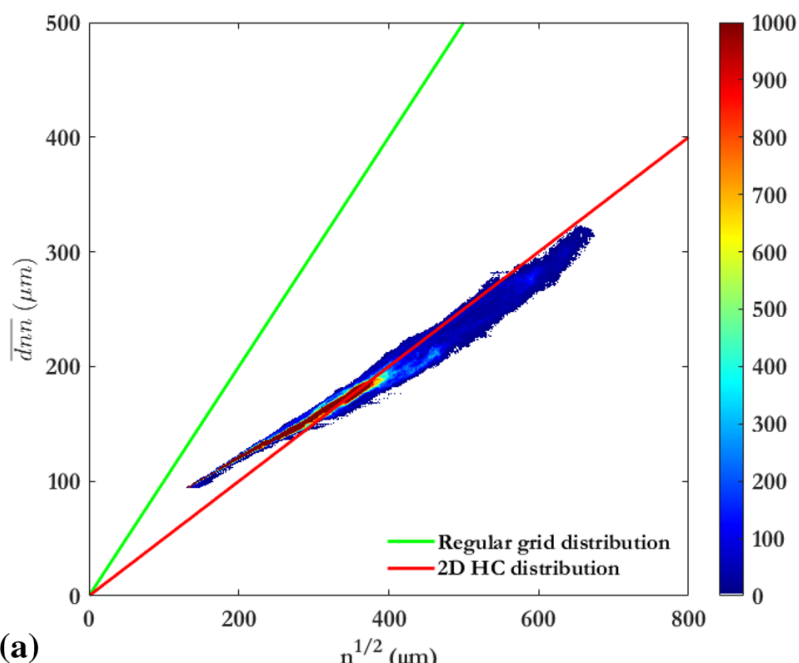

(a)

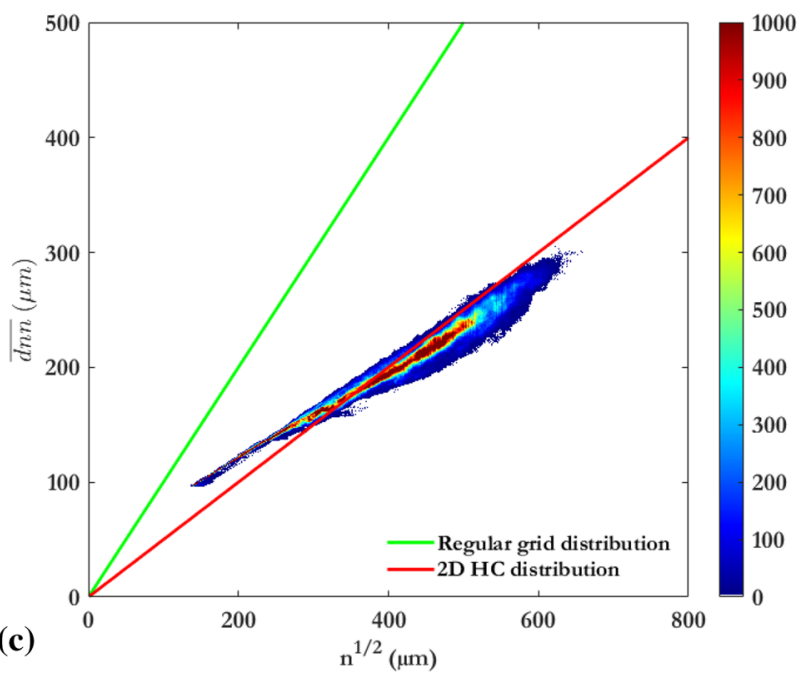

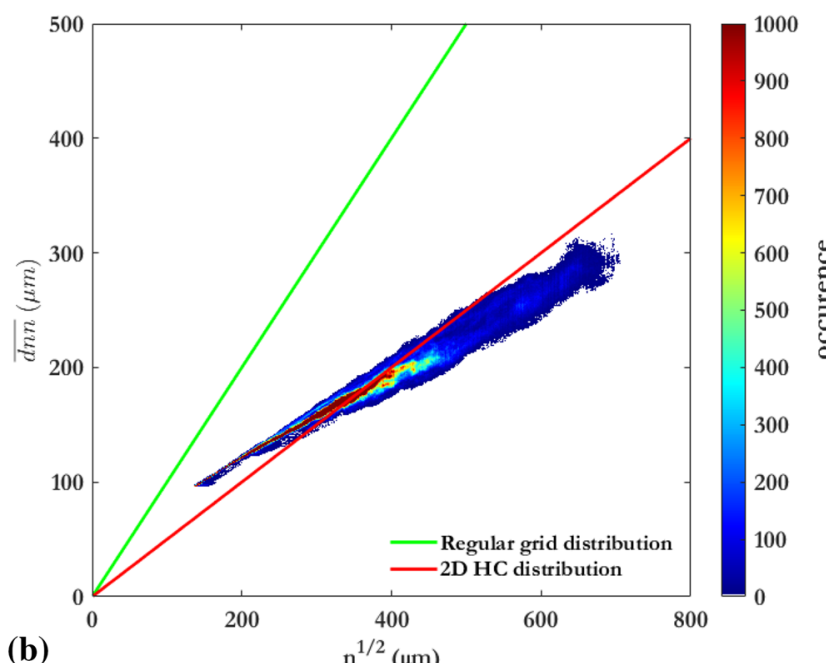

(b)

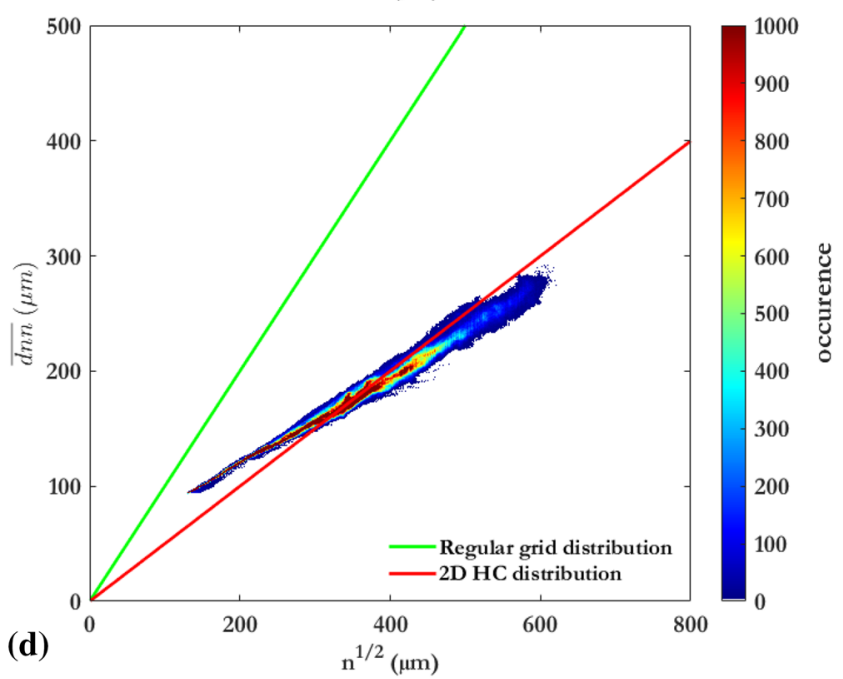

Fig. 12 dnn-droplet number density diagram for mean dnn values for non-reacting conditions, calculated with phase averaging at different phases a $0^{\circ}, \mathbf{b} 90^{\circ}, \mathbf{c} 180^{\circ}, \mathbf{d} 270^{\circ}$

metapixel, mean dnn behavior is related to mean droplet number density because droplet number density and dnn variations are complementary $\left(180^{\circ}\right.$ phase shift). As observed previously, mean dnn value increases when droplet number density value decreases and vice versa. Consequently, due to flow dynamics, phase averaging is clearly the better way to study data under non-reacting conditions because it captures the spray behavior, affected by flow dynamics. Results in the following section are thus presented only according to the phase-averaging method.

\subsection{Relationship between density, mean dnn and standard deviation of dnn}

Figures 12 and 13 present for four phases $\left(0^{\circ}, 90^{\circ}, 180^{\circ}\right.$ and $270^{\circ}$ ) the dnn-droplet number density diagrams, respectively, for the mean and the standard deviation of dnn values.
First, for all of the phases, $\overline{d n n}$ and $\sigma_{d n n}$ are linearly dependent upon the inverse square root of the mean droplet number density. The experimental scatter plots are always very close to the 2D Hertz-Chandrasekhar model predictions, but the measured $\alpha$ values are lower than under reacting conditions. It can be observed that, for all of the phases, when the inverse square root of the mean droplet number density is less than around $300 \mu \mathrm{m}$ (i.e., mean droplet number density around 1100 droplets per square centimeter), the majority of the scatter plot is located between both theoretical distributions for $d n n$ as well as for $\sigma_{d n n}$. On the contrary, when the inverse square root of the mean droplet number density is higher than $300 \mu \mathrm{m}$, the majority of the scatter plot is located below the 2D Hertz-Chandrasekhar distribution for $d n n$ and above it for $\sigma_{d n n}$. 


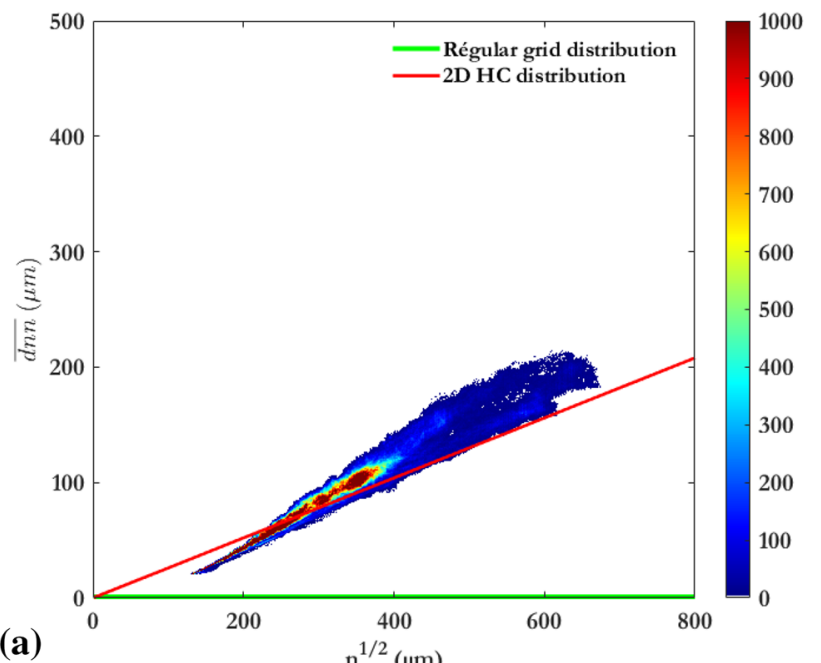

(a)

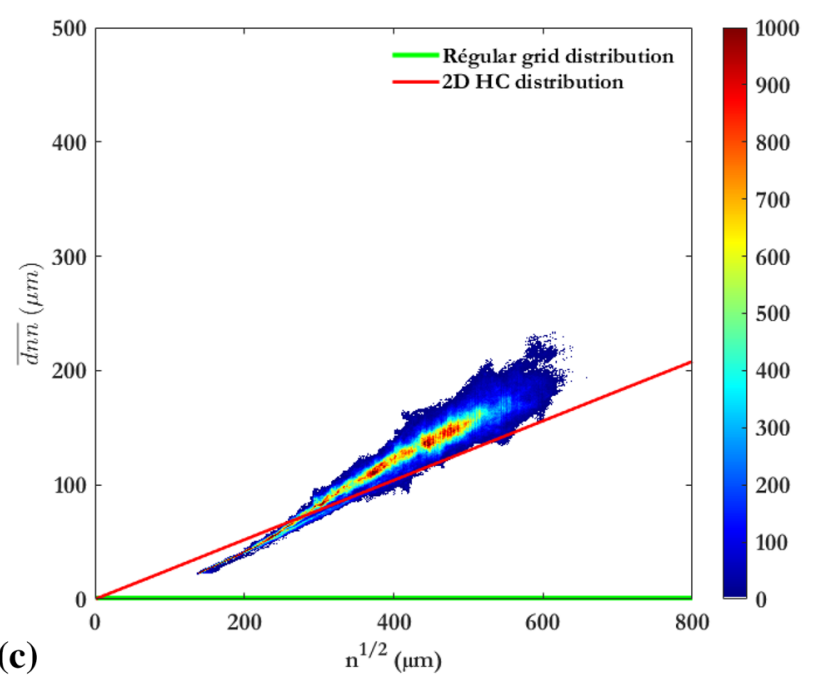

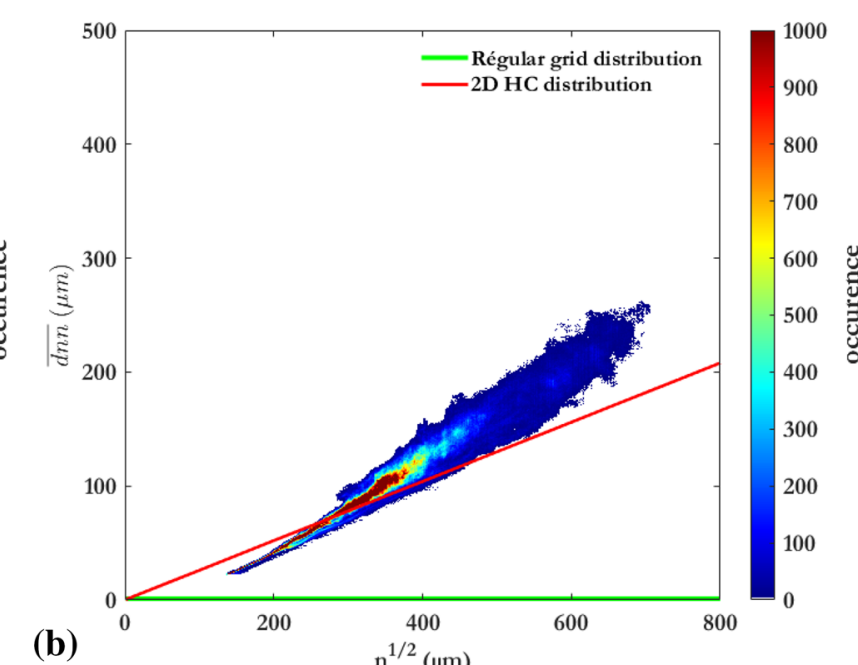

(b)

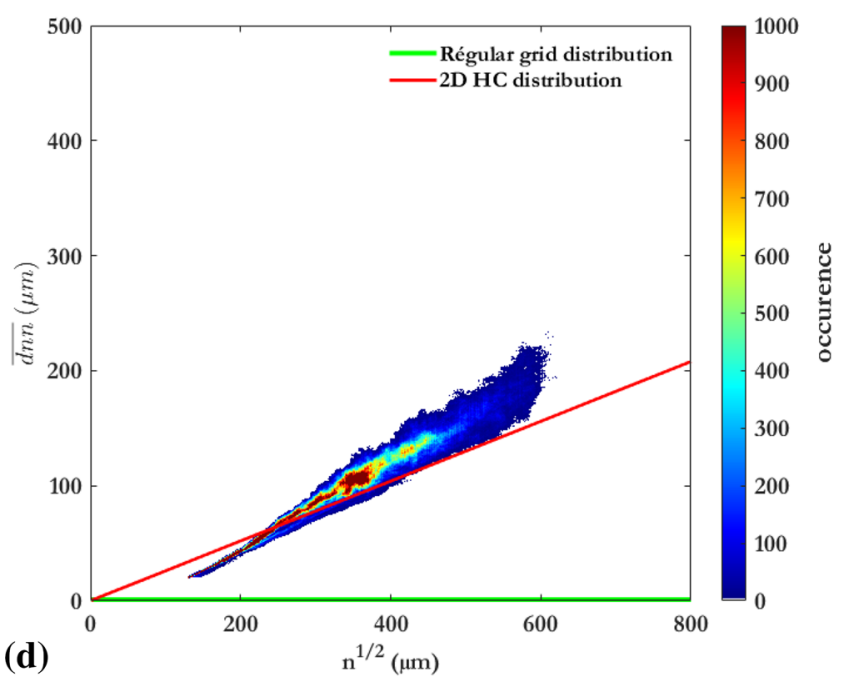

Fig. 13 dnn-droplet number density diagram for standard deviation dnn values for non-reacting conditions, calculated with phase averaging, at different phases $\mathbf{a} 0^{\circ}, \mathbf{b} 90^{\circ}, \mathbf{c} 180^{\circ}, \mathbf{d} 270^{\circ}$

The influence of the phase is negligible on the value of $\alpha$ : its mean value, close to 0.38 , varies by less than $6 \%$ with the phase (Fig. 14). Similarly, the mean value of $\beta$ is around 0.38 and the variation for each phase is less than $7 \%$. These results suggest that flow dynamics (presence of von Karmàn streets or not) does not affect the relationship between droplet number density and inter-droplet distance. Besides, for both evaporation and reacting conditions, results show that the $\alpha, \beta$ parameters are closer to the 2D Hertz-Chandrasekhar ones. Thanks to the analysis of Vicentini (2016), one can assume that the 3D Hertz-Chandrasekhar distribution is relevant to describe the droplet spatial distribution in the spray. Spray modeling could benefit from such an observation since current models are based on the regular grid model. However, the preferential segregation of droplets is not taken into account in that case (Vicentini 2016; Boutsikakis 2020). Finally, it is important to notice that, the experimental values of $\alpha$ and $\beta$ obtained in 2D may be influenced by the projection error (Vicentini 2016) due to the experimental process on one hand, and the preferential segregation on the other hand. Starting from DNS data, Boutsikakis (2020) and Vicentini (2016) have shown that the latter effect decreases the $\alpha$ parameter while it increases the $\beta$ parameter when the particle Stokes number decreases. Further experimental studies are needed to support this result.

\section{Conclusion}

Laser-based experiments performed on the PROMETHEE test rig were used to provide an experimental database for a two-phase flow under reacting and non-reacting conditions. Firstly, a Mie scattering image-processing algorithm was 


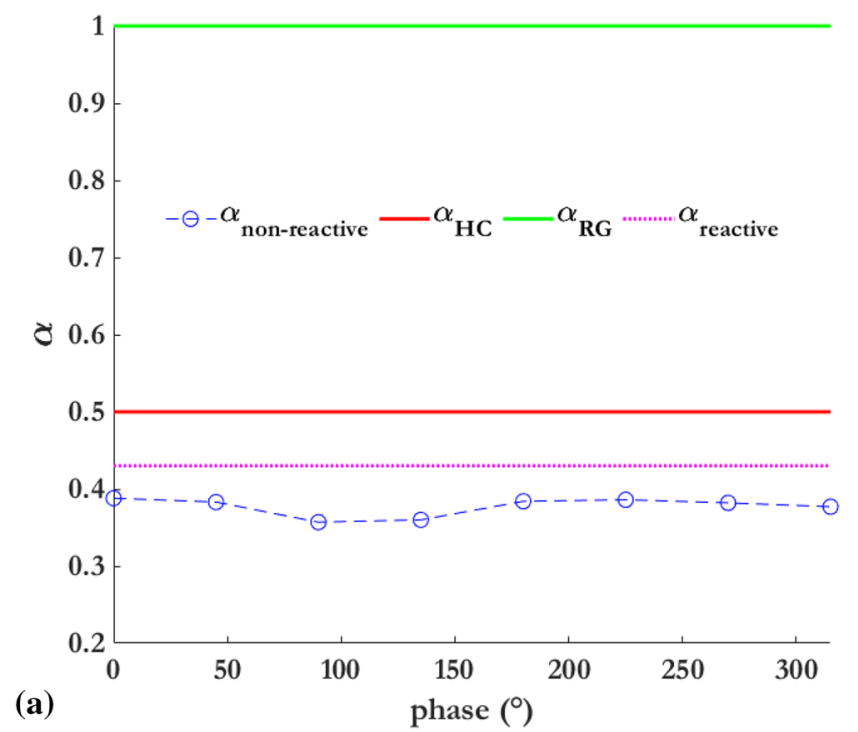

Fig. 14 Evolution of the $\mathbf{a}$ dimensionless mean $(\alpha)$ and $\mathbf{b}$ dimensionless standard deviation according to the phase $(\beta)$. The subscripts HC and RG correspond, respectively, to Hertz-Chandrasekhar and regu-

developed in order to locate droplets and calculate interdroplet distance. This method allowed to derive 2D maps of droplet number density, mean and standard deviation of the nearest-neighbor inter-droplet distance and the corresponding dnn-droplet number density diagrams. The droplet detection method may however be limited in the case of dense sprays, where significant blurring effects occur on the images due to multiple scattering, which can be reduced with structured illumination such as SLIPI. Secondly, under non-reacting conditions, a phase data averaging method was developed in order to capture the spray behavior affected by a von Karmàn vortex street shedding located downstream of the bluff-body. Results show that there is an affine relationship between the inverse square root of the droplet number density and the mean dnn values (resp. standard deviation dnn values) for both reacting and non-reacting conditions. Comparing the dimensionless mean and standard deviation values between experimental data and 2D Hertz-Chandrasekhar model indicates that spatial droplet distribution in the current spray is close to a uniform random distribution. This observation suggests that the current numerical models of evaporation and combustion of a fuel spray, which are generally based on the assumption of a simple regular grid distribution of the droplets in the spray, may be improved by using such a modeling approach.

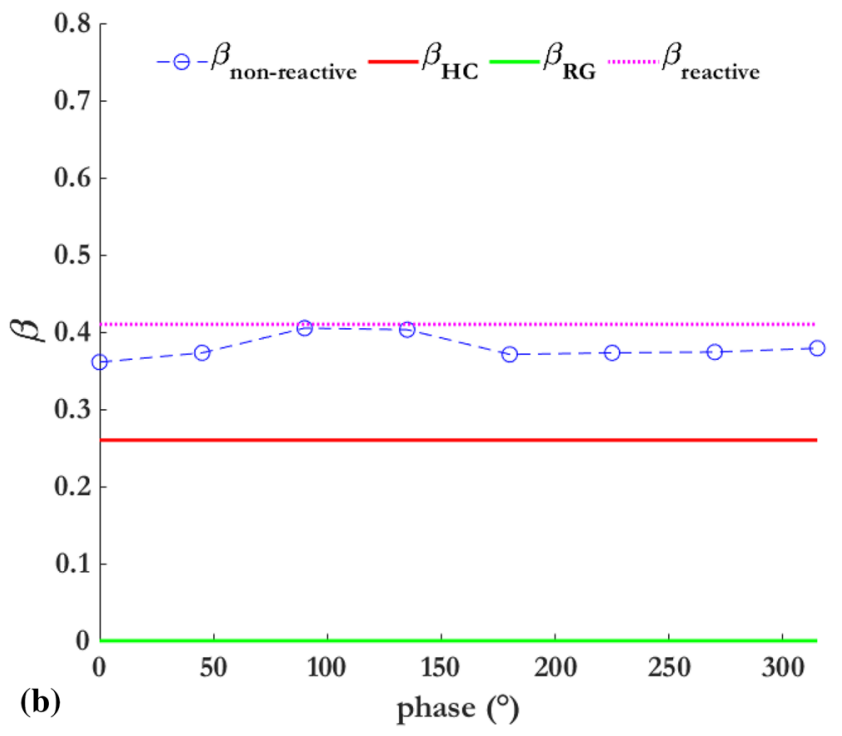

lar grid models. The subscripts non-reactive and reactive correspond to the values obtained from the measurements in non-reactive and reactive conditions

\section{Appendix}

Assuming that droplets are uniformly randomly distributed in a two-dimensional spray with a droplet number density $\bar{n}$, then, for a single droplet, the probability of having no droplet in a sphere (with a radius $r<d n n$ ) centered on this droplet can be estimated by the Poisson law:

$P(\lambda, k)=\frac{\lambda^{k}}{k !} \exp (-\lambda)$

where the parameter $k$ corresponds to the number of droplets enclosed in a surface and $\lambda$ is the product of the mean density and the surface. Considering a droplet, the probability $H\left(d n n_{i}\right)$ that its nearest-neighbor is located at a distance $d n n_{i}$ is equivalent to the probability of having no droplet enclosed in the surface $\pi * d n n_{i}^{2}$ times the probability of having no droplet between $d n n_{i}$ and $d n n_{i}+d\left(d n n_{i}\right)$. Thus, the probability is defined as:

$H\left(d n n_{i}\right)=2 \pi \bar{n} d n n_{i} * \exp \left(-\pi \bar{n} d n n_{i}^{2}\right)$

By definition, the mean value of the nearest-neighbor is:

$\overline{d n n_{i}}=\int_{0}^{\infty} d n n_{i} 2 \pi \bar{n} d n n_{i} \exp \left(-\pi \bar{n} d n n_{i}^{2}\right) d\left(d n n_{i}\right)$

Using the intermediate variable $u=\pi \bar{n} d n n_{i}^{2}$ and after some algebraic operations, the mean value expression is: 
$\overline{d n n_{i}}=\frac{1}{\sqrt{\pi}} \bar{n}^{-\frac{1}{2}} \Gamma\left(\frac{3}{2}\right)$

By definition, the variance of this variable is:

$$
\begin{aligned}
\operatorname{Var}\left(d n n_{i}\right) & =\int_{0}^{\infty}\left(d n n_{i}-\overline{d n n_{i}}\right)^{2} 2 \pi \bar{n} d n n_{i} \exp \left(-\pi \bar{n} d n n_{i}^{2}\right) d\left(d n n_{i}\right) \\
& =\frac{1}{\pi \bar{n}}\left[\Gamma(2)-\Gamma\left(\frac{3}{2}\right)^{2} \Gamma(1)\right.
\end{aligned}
$$

or, equivalently, using the standard deviation:

$\sigma_{d n n}=\frac{1}{\sqrt{\pi \bar{n}}}\left[\Gamma(2)-\Gamma\left(\frac{3}{2}\right)^{2} \Gamma(1)\right]^{\frac{1}{2}}$

These formulas use different values of the Gamma function: $\Gamma(1)=1, \Gamma\left(\frac{3}{2}\right)=\frac{\sqrt{\pi}}{2}$ and $\Gamma(2)=1$.

By replacing these values inside the relationships, we obtain Eq. 9.

For the three-dimensional case, the reader may refer to the article of Chandrasekhar (1943).

Acknowledgements Lola Rousseau is supported by a doctoral grant from ONERA and Région Occitanie.

Availability of data and material Not applicable.

Code availability Not applicable.

\section{Compliance with ethical standards}

Conflict of interest The authors declare no potential conflicts of interest with respect to the research, authorship, and/or publication of this article.

\section{References}

Abramzon B, Sirignano WA (1989) Droplet vaporization model for spray combustion calculations. Int J Heat Mass Transf 32(9):1605-1618. https://doi.org/10.1016/0017-9310(89)90043-4

Bailly P, Garreton D, Simonin O, Bruel P, Champion M, Deshaies B, Duplantier S, Sanquier S (1996) Experimental and numerical study of a premixed flame stabilized by a rectangular section cylinder. Combust Inst 26:923-929. https://doi.org/10.1016/S0082 -0784(96)80303-1

Boutsikakis A. (2020) Numerical simulation and physical analysis of the dispersion of charged inertial particles transported by stationary homogeneous isotropic turbulence. PhD Thesis, INPT.

Chandrasekhar S (1943) Stochastic problems in physics and astronomy. Rev Mod Phys. https://doi.org/10.1103/RevModPhys.15.1

Chauveau C, Halter F, Gökalp I (2006) Vaporization in threedimensional droplet arrays: effects of the fuel vapor saturation. ICLASS-2006, Kyoto, Japan. Paper ID ICLASS06-229

Chen G, Gomez A (1997) Dilute laminar spray diffusion flames near the transition from group combustion to individual droplet burning. Combust Flame 110:392-404. https://doi.org/10.1016/S0010 $-2180(97) 00087-4$
Chiu HH, Liu TM (1977) Group combustion of liquid droplets. Combust Flame 17:127-142. https://doi.org/10.1080/0010220770 8946823

Chiu HH, Kim HY, Croke EJ (1982) Internal group combustion of liquid droplets. $19^{\text {th }}$ symposium international on combustion. Combust Inst 19:971-980. https://doi.org/10.1016/S0082 -0784(82)80273-7

Cochet M, Bazile R, Ferret B, Cazin S (2009) Evaporation on polydispersed droplets in a highly turbulent channel flow. Exp Fluids 47:379-394. https://doi.org/10.1007/s00348-009-0667-9

Février P., Simonin O., and Legendre D. (2001) Particle dispersion and preferential concentration dependance on turbulent Reynolds number from direct numerical simulation and large eddy simulation of isotropic homogeneous turbulence. Proceedings of the fourth international conference on multiphase Flow, New Orleans, LA.

Godsave GAE (1953) Studies of the combustion of drops in a fuel spray: the burning of a single droplet of fuel. 4th symposium international on combustion. Combust Inst 4:818-830

Grosshans H, Kristensson E, Szasz R-Z, Berrocal E (2015) Prediction and measurement of the local extinction coefficient in sprays for 3D simulation/experiment data comparison. Int J Multiph Flow 72:218-232. https://doi.org/10.1016/j.ijmultiphaseflo w.2015.01.009

Hertz P (1909) Uber den gegenseitigen durchsnittlichen Abstand von Punkten, die mit bekannter mittlerer Dichte im Raume angeordnet sind. Math Ann 67(3):387-398

Imaoka RT, Sirignano WA (2005) Vaporization and combustion in a three-dimensional droplet arrays. Proc Combust Inst 30:19811989. https://doi.org/10.1016/j.proci.2004.08.049

Jiang TL, Chen WH, Tsai MJ, Chiu HH (1995) A numerical investigation of multiple flame configurations in convective droplet gasification. Combust Flame 3:221-238. https://doi.org/10.1016/00102180(95)92244-2

Kerstein AR, Law CK (1982) Percolation in combustion sprays I: transition from cluster combustion to percolate combustion in non-premixed sprays. $19^{\text {th }}$ symposium international on combustion. Combust Inst 19:961-969. https://doi.org/10.1016/S0082 $-0784(82) 80272-5$

Kostinski AB, Shaw RA (2001) Scale-dependent droplet clustering in turbulent clouds. J Fluid Mech 434:389-398. https://doi. org/10.1017/S0022112001004001

Labowski M (1976) The effects of nearest neighbor interactions on the evaporation rate of cloud particles. Chem Eng Sci 33:803-813. https://doi.org/10.1016/0009-2509(76)80054-1

Laven P. (2018) A computer program for scattering of light from a sphere using Mie theory and Debye series. V4.6.14 October 2018. http://www.philiplaven.com/mieplot.htm

Li SC, Libby PA, Williams FA (1993) Spray structure in counterflowing streams with and without a flame. Combust Flame 94:161177. https://doi.org/10.1016/0010-2180(93)90028-2

Lovett JA, Cross C, Lubarsky E, Zinn T (2011) A review of mechanisms controlling bluff-body stabilized flames with closely-coupled fuel injection. ASME Turbo Expo. https://doi.org/10.1115/ GT2011-46676

Mikami M, Mizuta Y, Tsuchida Y, Kojima N (2009) Flame structure and stabilization of lean-premixed sprays in a counterflow with low-volatility fuel. Proc Combust Inst 32:2223-2230. https://doi. org/10.1016/j.proci.2008.08.009

Mishra Y, Kristensson E, Berrocal E (2014) Reliable LIF/Mie droplet sizing in sprays using structured laser illumination planar imaging. Opt Express 22(4):4480-4492. https://doi.org/10.1364/ OE. 22.004480

Monchaux R, Bourgoin M, Cartellier A (2012) Analyzing preferential concentration and clustering of inertial particles in turbulence. 
Int J Multiph Flow 40:1-18. https://doi.org/10.1016/j.ijmultipha seflow.2011.12.001

Orain M, Mercier X, Grisch F (2005) PLIF imaging of fuel vapour spatial distribution in an acetone droplet stream, comparison with modeling. J Combust Sci Technol 177:249-278. https://doi. org/10.1080/00102200590900228

Réveillon J, Vervisch L (2005) Analysis of weakly turbulent dilutespray flames and spray combustion regimes. J Fluids Mech 537:317-347. https://doi.org/10.1017/S0022112005005227

Rouzaud O., Vicentini M. Lecourt R., Bodoc V., Simonin O. (2016) Experimental analysis of droplet spatial distribution in a spray burner. $27^{\text {th }}$ ILASS Conference.

Sahu S, Hardalupas Y, Taylor AMKP (2016) Droplet-turbulence interaction in a confined polydispersed spray: effect of turbulence on droplet dispersion. J Fluids Mech 794:267-309. https://doi. org/10.1017/jfm.2016.169

Sahu S, Hardalupas Y, Taylor AMKP (2018) Interaction of droplet dispersion and evaporation in a polydispersed spray. J Fluids Mech 846:37-81. https://doi.org/10.1017/jfm.2018.247

Silverman MA, Dunn-Rankin D (1994) Experimental investigation of a rectilinear droplet stream flame. J Combust Sci Technol 100:5773. https://doi.org/10.1080/00102209408935446

Sirignano WA (2014) Advances in droplet array combustion theory and modeling. Prog Energy Combust Sci 42:54-86. https://doi. org/10.1016/j.pecs.2014.01.002
Spalding DB (1953) The combustion of liquid fuels. 4th symposium international on combustion. Combust Inst 4:847-864

Squires KD, Eaton JK (1991) Preferential concentration of particles by turbulence. Phys Fluids A 3:1169. https://doi.org/10.1063/1.85804 5

Suzuki I., Chiu H. H. (1971) Multi-droplet combustion of liquid propellants. $9^{\text {th }}$ International Symposium on Space Technology and Science, p. 145

Vicentini M. (2016) Mise en évidence expérimentale et modélisation des régimes de combustion diphasique présents dans les foyers aéronautiques. $\mathrm{PhD}$ Thesis, ONERA.

Williams A (1973) Combustion of droplets of liquid fuels: a review. Combust Flame 21:1-31. https://doi.org/10.1016/00102180(73)90002-3

Publisher's Note Springer Nature remains neutral with regard to jurisdictional claims in published maps and institutional affiliations. 